\title{
TIPOS DE PLANTAS VASCULARES EN EL HERBARIO DE LA UNIVERSIDAD DE COSTA RICA (USJ)
}

\author{
Carlos O. Morales \& Natalie Villalobos T.
}

\begin{abstract}
An annotated checklist of the vascular plant types and ilustrations of types at the University of Costa Rica Herbarium (USJ) is presented in this paper. USJ has 215 type specimens in 47 families. Orchidaceae is the family with the greatest type number $(101,47 \%)$. Moreover, the herbarium has 105 type illustrations. In Orchidaceae two lectotypes are designated (for Myoxanthus vittatus and Trichocentrum costaricense) and a new combination is made (Echinosepala vittata, basionym: Myoxanthus vittatus).
\end{abstract}

RESUMEN: Se presenta una lista anotada de los tipos e ilustraciones de tipos de plantas vasculares del Herbario de la Universidad de Costa Rica (USJ). Son 215 tipos en 47 familias; el mayor número (101, 47\%) corresponde a Orchidaceae. Además, USJ posee 105 ilustraciones de tipos. En Orchidaceae se designan dos lectotipos (para Myoxanthus vittatus y Trichocentrum costaricense) y se hace una nueva combinación (Echinosepala vittata, basiónimo: Myoxanthus vittatus).

KeY wORds / PALABRAS ClAVE: Herbario USJ, tipos de plantas vasculares, vascular plant types, Costa Rica.

Desde la década de 1540 , cuando se preparó el primer herbario del mundo en el norte de Italia (Stafleu 1987), las colecciones de plantas secas han sido cruciales para el estudio de las especies vegetales. La información científica que puede proveer un herbario es no solamente variada, sino también sumamente valiosa, de modo que es posible realizar múltiples investigaciones en los herbarios (v.g. Ammann 1986): composición florística de regiones y países, variación morfológica de las especies según la distribución geográfica y altitudinal; cambios fenológicos a lo largo del año en diferentes regiones; impacto humano o natural en la historia fitogeográfica de una región o de un país; etnobotánica, con su amplio espectro que va desde el uso de nombres de plantas en la toponimia hasta la utilización de plantas maderables, ornamentales, alimenticias, tóxicas, medicinales y otros usos para obras artísticas, muebles, utensilios varios, armas, estructuras diversas y mucho más; estatus de conservación de especies poco conocidas (en este sentido, algunos investigadores llegan lejos, al proponer modelos matemáticos para deducir, a partir de datos de herbarios, si una especie puede considerarse extinta; véase Roberts \& McInerny 2003). Además, un herbario moderno posee, a menudo, una colección de ejemplares testigo de diversos tipos de investigaciones (agronómicas, químicas, farmacéuticas, etnobotánicas, fitogeográficas y de filogenia molecular).

La publicación de la lista de tipos de un herbario determinado (cf. Andrade et al. 1961, Clark et al. 2003, Lobo 2002, Nilsson \& Umaña 1995, Ruiz-Boyer \& González-Ball 2002) es importante por varias razones: 1) dilucida cuáles especímenes conforman la colección científica más valiosa del herbario, que es patrimonio científico tanto de la institución que alberga los especímenes como del país al que corresponde la diversidad allí representada; 2) es el mejor modo de divulgar el aporte del herbario al conocimiento florístico de determinadas regiones y países, y 3 ) contribuye a mejorar la comunicación entre botánicos y otros estudiosos de las plantas. En los últimos años también se han hecho esfuerzos para crear bases de datos con imágenes de las colecciones de tipos de algunos herbarios (Davies et al. 2002, Pupulin \& Romero-González 2003).

El Herbario de la Universidad de Costa Rica (USJ) fue fundado en 1931 en el Centro Nacional de Agricultura, que estaba ubicado en terrenos que a 
partir de 1940 ocupó esta universidad, en Montes de Oca, San José. Esta colección científica, con mayor edad que la propia universidad que la alberga, nació como una iniciativa personal del botánico y agrónomo José María Orozco. En las décadas posteriores del siglo XX, numerosas personas contribuyeron significativamente al crecimiento de las colecciones de plantas vasculares, hongos, briófitos, algas y líquenes de USJ. Es justo mencionar aquí los nombres de los máximos forjadores de la colección de plantas vasculares del Herbario USJ a lo largo de siete décadas: José María Orozco, Rafael Lucas Rodríguez, Luis Fournier Origgi (cuyo nombre tendrá pronto el Herbario USJ), Sergio Salas, Luis Poveda, Dora Emilia Mora y Jorge Gómez Laurito. Varias generaciones mantuvieron el interés y la persistencia necesarios para curar las colecciones y promover su desarrollo, incluso después del incendio habido en USJ en marzo de 1965 (L. Fournier, com. pers. 2001), que causó la pérdida irreparable de miles de especímenes, sobre todo de la colección del pionero Orozco. Fournier Origgi encabezó los esfuerzos para reanudar la recolecta y la formación de nuevas colecciones, aportando miles de ejemplares en las décadas de 1960 y 1970. Actualmente, USJ posee cerca de 90000 ejemplares, en su mayoría de plantas vasculares de Costa Rica. También, este herbario cuenta con material de Europa central, algunos países de América Central, EE.UU., México, Cuba, Jamaica y Argentina, entre otros. Además, se trabaja en una base de datos para registrar toda la información de los especímenes.

La lista anotada que se presenta aquí tiene la finalidad de dar a conocer los tipos, así como las fotografías, los dibujos y las fotocopias de tipos de plantas vasculares que se hallan en USJ. En este punto, consideramos importante referir que alrededor de 1995 tres isotipos de Orchidaceae fueron robados de USJ: Lepanthes ingramii Luer, Pleurothallis bitumida Luer y $P$. ingramii Luer. Al mismo tiempo desapareció el único ejemplar que el Herbario USJ tenía, en aquellos años, tanto de Sigmatostalix brownei Luer como de Zootrophion vulturiceps Luer. Este hecho insólito nos motiva a divulgar la colección de tipos, como una medida para protegerla.

La mayor parte de la información ha sido obtenida directamente de los especímenes tipo. Para obtener los datos de publicación hemos recurrido a las obras originales; cuando éstas no fueron accesibles, consultamos las bases de datos W3TROPICOS, del Jardín Botánico de Missouri, EE.UU., e IPNI (International Plant Names Index). En un futuro cercano esta información será accesible en Internet, donde podremos realizar las actualizaciones pertinentes y, en lo posible, agregar ilustraciones de los tipos de USJ. Esperamos que la información proporcionada aquí sea interesante y útil sobre todo para los estudiosos de las floras neotropicales.

En la lista, los táxones mayores (divisiones y clases) se ordenan en secuencia evolutiva según el sistema de Cronquist (1992). Para mayor facilidad de uso, las familias de cada clase se ordenan alfabéticamente. Así, tenemos dos divisiones: 1) Pteridophyta, solamente con las clases Lycopodiopsida y Polypodiopsida, y 2) Spermatophyta, con dos clases: Dicotyledoneae y Monocotyledoneae. Los datos de cada tipo se ofrecen según el siguiente formato: [Número (consecutivo en cada familia). Nombre científico (en la publicación original) autor(es), publicación. País: provincia o estado; cantón (si se conoce), lugar, coordenadas (cuando se ofrecen en la etiqueta de herbario), altitud (en metros, raro en pies), fecha, recolector con número (herbarios donde se hallan los tipos según la literatura; en algunos casos según observación personal del primer autor, C.O.M.). Otros nombres del mismo taxon y (cuando pertinente) alguna nota adicional]. El número de herbario de USJ solamente se ha agregado cuando falta un número de recolecta. Las abreviaturas usadas son: ca., circa, CT, clonotipo, f, figura(s), fl, flores, fr, frutos, ft., feet $=$ pies de altitud (en inglés), HT, holotipo, ILT, isolectotipo, IT, isotipo, LT, lectotipo, m, metros de altitud, PT, paratipo, s.d. (sine dato, sin fecha), s.n., sin número, $\mathrm{ST}$, sintipo. Aquí queremos mencionar que USJ posee varios clonotipos en Orchidaceae (p.ej., Chondrorhyncha lankesteriana Pupulin, Epidendrum candelabrum Hágsater, Pleurothallis compressa Luer, Pleurothallis scaphipetala Luer, Stelis morae Luer, Trichosalpinx lankesteriana Luer); este término se ha vuelto común en la literatura actual para los ejemplares preparados, a posteriori, a partir de la misma planta cultivada de la que se preparó el holotipo. Sin embargo, es claro que no se trata de una categoría oficial de tipo, por cuanto no se cita en la publicación original del nombre respectivo ni se 
menciona en el Código Internacional de Nomenclatura Botánica (Greuter et al. 2000).

Las cifras totales indican que USJ posee 215 tipos y 105 ilustraciones de tipos (fotos, fotocopias y dibujos) en 47 familias de plantas vasculares. Más detalladamente, se trata de 68 HT, 97 IT, 48 PT, 2 LT, 72 fotos, 29 fotocopias y 4 dibujos. Con 101 tipos, la familia Orchidaceae abarca el $47 \%$ de los tipos de USJ: son 55 HT, 27 IT, 17 PT y 2 LT. El número notablemente alto de tipos de orquídeas refleja una gran actividad orquideológica en USJ desde la década de 1960. Además, es interesante que en USJ se hallan 17 ejemplares tipo en líquido; 15 de éstos son Orquidáceas. Por otro lado, la familia Araliaceae comprende el 65\% (47) de las fotos de tipos de este herbario.

\section{División PTERIDOPHYTA}

(helechos y afines)

\section{Clase LYCOPODIOPSIDA}

\section{ISOËTACEAE}

1. Isoëtes savannarum L.D. Gómez, Phytologia 49(4): 339. 1981. Costa Rica: Guanacaste; inmediationem Crucis, prope loco Tanques dicto, ca. 200 m., L.D. Gómez 17350 (HT: CR, IT: US, F, NY, MO, BM, K). Un ejemplar anotado como isotipo en USJ, pero los detalles de la localidad de éste difieren de los anteriores: "En charcas semiestacionales de sabana. $1.5 \mathrm{~km}$ antes de La Cruz, Guanacaste, 25 m, agosto 1981, L.D. Gómez 7350". Guanacaste; $1,5 \mathrm{~km}$ al sur de La Cruz, ca. 210 m, 22 ago 1981, J. Gómez Laurito 7088 (PT: USJ) = Isoëtes panamensis Maxon \& C.V. Morton, Ann. Missouri Bot. Gard. 26(4): 272. 1939.

2. Isoëtes tryoniana L.D. Gómez, Rev. Biol. Trop. 17(1): 108, f.8. 1972. Costa Rica: San José; Cordillera de Talamanca; lago mayor del macizo del Chirripó Grande, $3300 \mathrm{~m}$, con otra isoetácea (I. storkii Palmer), L.D. Gomez PtC-1032 (HT: USJ). El ejemplar en USJ tiene datos ligeramente distintos: "Colectado en Lago Mayor, Valle de las Morenas, Macizo de Chirripó Grande, 3800 m, 6-2-67, Colec. Luis D. Gómez" (USJ-5594).
En USJ, también un ejemplar en líquido anotado como isotipo: Lago Mayor, Páramo de Chirripó Grande, 3400 m, 6 febr 1967, Luis D. Gómez P. (USJ-84440). = Isoëtes storkii T.C. Palmer, Amer. Fern J. 22(4): 136. 1932.

\section{Clase POLYPODIOPSIDA (= Filicopsida)}

\section{LOMARIOPSIDACEAE}

1. Elaphoglossum nanum A.F. Rojas, Brenesia 4748: 10. 1997. Costa Rica: Puntarenas; Parque Nacional Chirripó, Refugio Los Crestones, 31 jul 1987, G. Vargas \& E. Arévalo 370 (HT: CR, IT: INB, NY, USJ). USJ in litter., non vidimus.

\section{División SPERMATOPHYTA (plantas seminíferas)}

\section{Clase MAGNOLIOPSIDA \\ (= Dicotyledoneae)}

\section{ACANTHACEAE}

1. Herpetacanthus stenophyllus Gómez-Laur. \& Grayum, Novon 1(1): 15. 1991. Costa Rica: Limón; Cordillera de Talamanca, Reserva Biológica Hitoy Cerere, siguiendo el Sendero Espavel hasta la fila, $9^{\circ} 39^{\prime} 15^{\prime \prime} \mathrm{N}, 83^{\circ} 01^{\prime} 20^{\prime \prime} \mathrm{W}, 695 \mathrm{~m}, 16$ febr 1989 (fl), G. Herrera \& A. Chacón 2404 (HT: CR, IT: F, MO, USJ).

2. Justicia bitarkarae Gómez-Laur., Brenesia 33: 140. 1990 [1991]. Costa Rica: Limón; Cordillera de Talamanca, Reserva Biológica Hitoy Cerere, cuenca superior del Río Hitoy, subiendo al Cerro Bitarkara, 09 $38^{\prime} 25^{\prime \prime} \mathrm{N}, 83^{\circ} 07^{\prime} 20^{\prime \prime} \mathrm{W}, 500 \mathrm{~m}, 27$ febr 1989 (fl, fr), G. Herrera \& M. Solís 2469 (HT: CR, IT: F, MO, USJ). Dos isotipos en USJ.

3. Justicia peninsularis Gómez-Laur. \& Hammel, Novon 4(4): 355. 1994. Costa Rica: Puntarenas; Osa, Península de Osa, Aguabuena de Rincón, $8^{\circ} 42^{\prime} 40^{\prime \prime} \mathrm{N}, 83^{\circ} 31^{\prime} 40^{\prime \prime W}, 400 \mathrm{~m}, 25$ oct 1990, $\mathrm{G}$. Herrera 4505 (HT: CR, IT: ARIZ, INB, MO, US). Un isotipo en USJ.

4. Kalbreyeriella rioquebradasiana Gómez-Laur. \& Hammel, Novon 4(4): 357. 1994. Costa Rica: 
San José; Cerro de la Muerte, Pérez Zeledón, km 115-116, Carretera Interamericana sur, $9^{\circ} 28^{\prime} 40^{\prime \prime} \mathrm{N}, 83^{\circ} 41^{\prime} 25^{\prime \prime} \mathrm{W}, 1750 \mathrm{~m}, 4$ febr 1991 , B. Hammel et al. 18053 (HT: CR, IT: ARIZ, CAS, F, INB, MO, US, USJ).

\section{ANNONACEAE}

1. Guatteria tonduzii Diels var. leptopus R.E. Fr., Acta Horti Berg. 12(3): 357. 1939. Costa Rica: Puntarenas; Cañas Gordas, 1100 m, mar 1897 (fl, fr), H. Pittier 10958 (HT: M, IT: BR, C, US). USJ: Foto del holotipo ex M.

APIACEAE (= Umbelliferae)

1. Hydrocotyle x nubigena R.L. Rodr., Rev. Biol. Trop. 8(1): 69. 1960. (H. mexicana Cham. \& Schltdl. x H. pusilla A. Rich.). Costa Rica: San José; Vásquez de Coronado, camino de las $\mathrm{Nu}-$ bes a Bajo de la Rosa, 28 jul 1957 (fl), R.L. Rodríguez 508 (HT: USJ).

2. Myrrhidendron chirripoënse Suess., Bot. Jahrb. System. 72(2): 280. 1942. Costa Rica: San José; Pérez Zeledón, Chirripó Grande, 3800 m, 28 abr 1932 (fl), W. Kupper 1174 (HT: M). Un fragmento del tipo (IT) en USJ, que perteneció a F. Gutiérrez Braun, con dibujos y una fotografía de H. Weber, marzo de 1957.

3. Triphylleion chirripoi Suess., Bot. Jahrb. Syst. 72(2): 279. 1942. Costa Rica: San José; Pérez Zeledón, Cerro Chirripó Grande, 3450 m, 29 abr 1932, W. Kupper 1180 (HT: M). Un fragmento del tipo (IT) en USJ. Niphogeton chirripoi (Suess.) Mathias \& Constance, Brittonia 14(2): 154. 1962.

\section{APOCYNACEAE}

1. Fernaldia pandurata (A. DC.) Woodson var. glabra Ant. Molina, Ceiba 3(2): 95. 1952. Honduras: Cortés; "Loroco", faldas de la Montaña Santa Ana, 210 m, 6 dic 1950 (fl), A. Molina 3640 (HT: EAP, IT: F). Un isotipo en USJ.

\section{ARALIACEAE}

En esta familia USJ posee fotografías en blanco y negro de tipos de Berlín (B), Copenhague (C) y Múnich (M), que fueron traídas por el Dr. Rafael Lucas Rodríguez del Herbario de la Universidad de California en Berkeley (UC). Se trata de copias de fotos tomadas por el Field Museum of Natural History, de Chicago, EE.UU., con apoyo económico de la Fundación Rockefeller.

1. Aralia ferruginea Kunth, Nov. Gen. Sp. 5: 7. 1821. Ecuador: Loja; crescit in temperatis Regni Quitensis, inter Paramo de Saraguru et Ona, alt. 1200 hex [ca. $2400 \mathrm{~m}$ ], floret julio [sine anno], Humboldt \& Bonpland s.n. (B). Schefflera ferruginea (Kunth) Harms in Engl. \& Prantl, Nat. Pflanzenfam. 3(8): 36. 1894.

2. Aralia laetevirens Gay, Fl. Chil. 3: 151. 1848. Chile; s.d., Gay 70 (B). Pseudopanax laetevirens (Gay) H. Baillon, Adansonia 12: 148. 1878. Didymopanax laetevirens (Gay) Seem. (no se hallaron datos de publicación de este nombre, que aparece en la etiqueta del ejemplar).

3. Didymopanax angustissimum E. Marchal, Fl. Br. (Martius) 11(1): 241. 1878. Brasil: "In sylvis ... \& Serra Estrella", oct 1823, Riedel s.n. (M).

4. Didymopanax cordatum Taub., Bot. Jahrb. Syst. 17: 509. 1893. Brasil, s.d., Glaziou 19413 (C).

5. Didymopanax longipetiolatum E. Marchal, Fl. Bras. (Martius) 11(1): 234. 1878. Brasil; s.d., Dr. Pohl "herb. 5368" (M).

6. Didymopanax macrocarpum Seem., J. Bot. 6: 132. 1868. Brasil; s.d., Martius s.n. (M).

7. Didymopanax nebularum Harms, Notizbl. Bot. Gart. Berlin-Dahlem 11: 293. 1931. Bolivia: Negracota, 3500 m, 6 mar 1928, C. Troll 1803 (B).

8. Didymopanax pachycarpum E. Marchal, Fl. Bras. (Martius) 11(1): 236. 1878. Brasil; s.d., Sello 361 (B).

9. Didymopanax spruceanum Seem., J. Bot. 6: 132. 1867. Brasil; s.d., R. Spruce 2307 (HT: K). Foto de un isotipo ex B. Schefflera spruceana (Seem.) Maguire, Steyerm. \& Frodin, Mem. New York Bot. Gard. 38: 69. 1984.

10. Didymopanax vinosum $\mathrm{E}$. Marchal var. attenuatum E. Marchal, Fl. Bras. (Martius) 11(1): 239. 1878. Brasil; s.d., Martius s.n. (M).

11. Didymopanax weberbaueri Harms, Bot. Jahrb. 
Syst. 42(1): 161. 1908. Perú; s.d., A. Weberbauer 4694 (B).

12. Gilibertia caucana Harms, Repert. Spec. Nov. Regni Veg. 23(18-25): 300. 1927. Colombia: Cauca; "wächst in dichten parkartigen Wäldern an den Rändern des Cauca-Thal", 800-1400 m, Juli (sine anno), F.C. Lehmann 4733 (B). Dendropanax caucanus (Harms) Harms, Notizbl. Bot. Gart. Berlin-Dahlem 15(5): 692. 1942.

13. Gilibertia heterophylla E. Marchal, F1. Bras. (Martius) 11(1): 246. 1878. Brasil; s.d., Glaziou 3021 (C).

14. Gilibertia langsdorfii E. Marchal, Fl. Bras. (Martius) 11(1): 248. 1878. Brasil; "in sylvis mont. prope Itatiaia \& Serra Estrella", s.d., Riedel 1269 (C).

15. Gilibertia rothschuhii Harms, Bot. Jahrb. Syst. 23: 126. 1896. Nicaragua; s.d., Rothschuh 112 (B). = Dendropanax arboreus (L.) Decne. \& Planch., Rev. Hort., ser. 4, 3: 107. 1854.

16. Gilibertia samydifolia $\mathrm{E}$. Marchal in Urb., Symb. Antill. 1: 201. 1899. Panamá; ago 1862, S. Hayes $748(\mathrm{M})$. = Dendropanax arboreus (L.) Decne. \& Planch. (vide supra).

17. Oreopanax allocophyllus Harms ["alloeophyllus" en IPNI], Repert. Spec. Nov. Regni Veg. 23: 299. 1927. Perú; s.d., A. Weberbauer 6680 (B).

18. Oreopanax angulare Kunth, J. Bot. 3: 276. 1865. Sudamérica (Am. austr.), s.d., Humboldt s.n. in Herb. Willdenow (B).

19. Oreopanax apurimacensis Harms, Notizbl. Bot. Gart. Berlin-Dahlem 11: 288. 1931. Perú; s.d., Raimondi 12514 (B).

20. Oreopanax aquifolium Harms, Bot. Jahrb. Syst. 42(1): 156. 1908. Perú; 11 jul 1903, A. Weberbauer 3350 (B).

21. Oreopanax crataegodorus Harms, Notizbl. Bot. Gart. Berlin-Dahlem 11: 288. 1931. Colombia; 7 mar 1928, A. Schultze 1279 (B).

22. Oreopanax dombeyanum Decne. \& Planch., Rev. Hortic. Ser. 4(3): 108. 1854 (nomen, fide IPNI). Perú; 1778-1788, Dombey s.n. (B).

23. Oreopanax cheirophyllum Seem., J. Bot. 3: 275. 1865. Sudamérica (“Am. austr.”); s.d., Humboldt in Herb. Willdenow (B).

24. Oreopanax eriocephalus Harms, Bot. Jahrb. Syst. 20(3, Beibl. 49): 67. 1895. Ecuador:
Cuenca; 2500-3000 m, ago 1888, F.C. Lehmann 7317 (B).

25. Oreopanax kuntzei Harms, Rev. Gen. Bot. 3(2): 116. 1898. Bolivia; 1000 m, 13-21 abr 1892, O. Kuntze s.n. (B).

26. Oreopanax raimondii Harms, Notizbl. Bot. Gart. Berlin-Dahlem 11: 290. 1931. Perú: La Libertad, "bajada del tito de la Sopapilla a la Capellanía y camino a Condormarca", s.d., A. Raimondi 12841 (B).

27. Oreopanax reticulatum Decne. \& Planch. ex Seem., J. Bot. 3: 275. 1865. Venezuela; s.d., Humboldt in Herb. Willdenow (B). O. reticulatum Decne. \& Planch., Rev. Hortic. Ser. 4(3): 108. 1854, nomen.

28. Oreopanax schultzei Harms, Notizbl. Bot. Gart. Berlin-Dahlem 11: 291. 1931. Colombia; Sierra Nevada de Santa Marta, 2200 m, 1928, A. Schultze 1103 (B).

29. Oreopanax trollii Harms, Notizbl. Bot. Gart. Berlin-Dahlem 11: 292. 1931. Bolivia; 1928, C. Troll 2724 (B).

30. Schefflera dolichostyla Harms, Bot. Jahrb. Syst. 42(1): 152. 1908. Perú; s.d., A. Weberbauer 735 (B).

31. Schefflera euryphylla Harms, Bot. Jahrb. Syst. 42(1): 151. 1908. Perú; 2700 m, 20 ene 1903, A. Weberbauer 2288 (B).

32. Schefflera herzogii Harms, Meded. Rijks-Herb. 29: 6. 1916. Bolivia; s.d., Herzog 2270 (B).

33. Schefflera inambarica Harms, Bot. Jahrb. Syst. 42(1): 150. 1908. Perú; s.d., A. Weberbauer 1080 (B).

34. Schefflera lehmannii Harms, Bot. Jahrb. Syst. 20 (Beibl. 49): 69. 1895. Colombia; Las Juntas del Dagua, s.d., F.C. Lehmann 7732 (B). = Schefflera sphaerocoma (Benth.) Harms, Nat. Pflanzenfam. 3(8): 37.1894 [1898].

35. Schefflera microcephala Harms, Bot. Jahrb. Syst. 42: 148. 1908. Perú; s.d., A. Weberbauer 2108 (B). = Schefflera sprucei (Seem.) Harms, Nat. Pflanzenfam. 3(8): 36. 1894.

36. Schefflera minutiflora Harms, Bot. Jahrb. Syst. 42: 153. 1908. Perú: Loreto; montes prope Moyobamba, 1500-1600 m, sept 1904, A. Weberbauer 4735 (B).

37. Schefflera monzonensis Harms, Bot. Jahrb. 
Syst. 42: 154. 1908. Perú: Huánuco; Huamalies, prope Monzón, 2100-2500 m, jul 1903, A. Weberbauer 3418 (B).

38. Schefflera moyobambae Harms, Engl. Jahrb. 42: 154. 1908. Perú: Loreto; prope Moyobamba orient versus, 1300-1400 m, sept 1904, A. Weberbauer 4741 (B). Dos fotos del tipo (en dos láminas).

39. Schefflera sodiroi Harms, Repert. Spec. Nov. Regni Veg. 15: 246. 1918. Ecuador; 2000-3200 m, s.d., A. Sodiro 619 (B).

40. Schefflera tipuanica Harms, Notizbl. Bot. Gart. Berlin-Dahlem 11: 287. 1931. Bolivia: Hacienda Simaco sobre el camino a Tipuani, $1400 \mathrm{~m}$, febr 1920, O. Buchtien 5509 (B).

41. Schefflera trollii Harms, Notizbl. Bot. Gart. Berlin-Dahlem 11: 287. 1931. Bolivia; Negracota, ca. 3200 m, 6 mar 1928, C. Troll 1781 (B).

42. Schefflera ulei Harms, Verh. Bot. Vereins Prov. Brandenburg 47: 186. 1905. Perú: Loreto; Cerro de Escaler, 1500 m, E. Ule 6761 (B).

43. Schefflera viguieriana Harms, Bot. Jahrb. Syst. 42: 150. 1908. Perú; 1903, A. Weberbauer 2113 (B). = Schefflera angulata (Ruiz \& Pav.) Harms, Nat. Pflanzenfam. 3(8): 36. 1894.

44. Schefflera weberbaueri Harms, Bot. Jahrb. Syst 42: 151. 1908. Perú; jul 1903, A. Weberbauer 3413 (B). = Schefflera angulata (Ruiz \& Pav.) Harms (vide supra).

45. Schefflera yuncacoyae Harms, Bot. Jahrb. Syst 42: 155. 1908. Perú; 8 jun 1902, A. Weberbauer 1153 (B). = Schefflera patula (Rusby) Harms, Nat. Pflanzenfam. 3(8): 37. 1894.

46. Sciadophyllum japurense Mart. \& Zucc. ex Marchal, Fl. Bras. (Martius) 11(1): 244, Taf. 69. 1878. Brasil: Pará; Araracoara, s.d., Martius s.n. (M). Schefflera japurensis (Mart. \& Zucc.) Harms, Nat. Pflanzenfam. 3(8): 38. 1894.

\section{ARISTOLOCHIACEAE}

1. Aristolochia gorgona M.A. Blanco, Brittonia 54(1): 31, f.1-2. 2002. Costa Rica: Heredia; Puerto Viejo de Sarapiquí, Estación Biológica La Selva, Quebrada Surá, cerca del Laboratorio, $10^{\circ} 26^{\prime} \mathrm{N}, 83^{\circ} 59^{\prime} \mathrm{W}, 45 \mathrm{~m}, 4$ ene 2001, M. Blanco 1752 (HT: USJ, pro parte en líquido: ginostemio y segmento del limbo, IT: CR, MO, NY).

\section{ASCLEPIADACEAE}

1. Gonolobus tenuisepalus Krings, Sida 20(1): 105, f.1. 2002. Costa Rica: Puntarenas; Coto Brus, San Vito, Estación Biológica Las Cruces, 1200 m, 11 mar 1997, A. Krings 274 (HT: F, IT: CR, NCSC, US). Un isotipo en USJ.

\section{ASTERACEAE}

1. Roldana scandens Poveda \& Kapelle, Brenesia 37: 157. 1992. Costa Rica: San José; Cordillera de Talamanca, Dota, Copey, Reserva Forestal Los Santos, por el camino hacia San Gerardo, cerca del caserío de Jaboncillos, 2900 m, 26 ene 1992 (fl), M. Kappelle 5843 (HT: CR, IT: ASD, COL, F, MEXU, MO, NY, U, US). Dos isotipos en USJ.

2. Verbesina tapantiana Poveda \& Hammel, Brenesia 32: 123. 1989. Costa Rica: Cartago; Parque Nacional Tapantí, por la orilla del Río Grande de Orosi, 1200 m, 24 oct 1989, B. Hammel et al. 17892 (HT: CR, IT: MO). Un isotipo en USJ.

\section{BOMBACACEAE}

1. Matisia tinamastiana A. Estrada \& Cascante, Brenesia 49-50: 80, f.1-2. 1998. Costa Rica: San José; Pérez Zeledón, Tinamaste, Finca Tinamaste (17 km de San Isidro en camino a Dominical), Fila Tinamaste, 650 m, 24 mar 1998, A. Estrada et al. 1484 (HT: CR, IT: USJ). Loc. cit., 650-700 m, 13 jun 1999, A. Estrada et al. 1611 (PT: USJ).

2. Quararibea aurantiocalyx W.S. Alverson, Brittonia 41(1): 68. 1989. Costa Rica: Puntarenas; Coto Brus, alrededores de Cerro Las Cruces, San Vito de Java, 18 ago 1967, S. Salas 703 (HT: USJ, IT: WIS).

3. Quararibea gomeziana Alverson, Brittonia 41(1): 61. 1989. Costa Rica: Limón; Río Sand Box, ca. $0.5 \mathrm{~km}$ upstream from Bribri-Cahuita rd. crossing, 09 $38^{\prime} 47^{\prime \prime} \mathrm{N}, 82^{\circ} 49^{\prime} 28^{\prime \prime} \mathrm{W}, 90 \mathrm{~m}, 28$ oct 1983, W. Alverson 2136 (HT: WIS, IT: B, BR, COL, DUKE, F, G, GH, HUA, K, MO, NY, PMA, S, UC, US, USJ, Z). 


\section{CAMPANULACEAE}

1. Burmeistera cyclostigmata Donn. Sm., Bot. Gaz. 20(7): 291. 1895. Costa Rica: Santa Clara; Suerre, 900 ft. [275 m], Febr 1896, J. Donnell Smith 6623 (ST: M). USJ: Foto ex M.

2. Burmeistera quercifolia Gómez-Laur. \& L.D. Gómez, Brenesia 22: 352. 1984. Costa Rica: Alajuela; Zarcero, Cerros Volcán Viejo, ca. 2200 m, 11 nov 1983, J. Gómez Laurito 9677 (PT: USJ).

3. Centropogon nubicola Gómez-Laur. \& L.D. Gómez, Phytologia 51(7): 477. 1982. Costa Rica: Alajuela; Cerro Las Nubes, Volcán Miravalles, 1600 m, 9 abr 1982. I.A. Chacón 74 (PT: USJ).

\section{CECROPIACEAE}

1. Coussapoa parviceps Standl., Proc. Biol. Soc. Washington 37: 51. 1924. Costa Rica: Puntarenas; Valle de Agua Buena, Cañas Gordas, 1100 m, febr 1897, H. Pittier 11166 (HT: US, IT: MO). USJ: Foto y fotocopia de un isotipo ex M.

\section{CHRYSOBALANACEAE}

1. Licania riverae Prance, Kew Bull. 50(4): 711. 1995. Costa Rica: Guanacaste; Parque Nacional Rincón de la Vieja, San Jorge, 1044'N, 85¹7'W, 600 m, 7 mayo 1991, G. Rivera 1296 (HT: K, IT: INB, MO). Un isotipo en USJ.

\section{COMBRETACEAE}

1. Buchenavia costaricensis Stace, Novon 5(1): 107. 1995. Costa Rica: Puntarenas; Osa, Península de Osa, Reserva Forestal Golfo Dulce, Aguabuena, sector cuenca oeste, $8^{\circ} 42^{\prime} 02^{\prime \prime} \mathrm{N}$, 8331'30"W, 50-150 m, 28 sept 1991, R. Aguilar 502 (HT: MO, IT: CR, LTR). Un isotipo en USJ.

\section{DICHAPETALACEAE}

1. Stephanopodium costaricense Prance, Kew Bull. 50(2): 300. 1995. Costa Rica: Puntarenas, Osa, Sierpe, Península de Osa, Fila Banegas, Rancho Quemado, 841’N, 83³3’W, 400 m, 8 mayo 1992 (fl), J. Marín 489 (HT: K, IT: CR, MO, WAG). Un isotipo en USJ. Esta extraña especie fue determinada al principio como Tapura Aubl.

\section{EUPHORBIACEAE}

1. Croton megistocarpus J.A. González \& Poveda, Lankesteriana 8: 9. 2003. Costa Rica: Guanacaste; Tilarán, rumbo a Tierras Morenas, 8 ago 1973, L. Poveda 603 (PT: USJ). Guanacaste; Parque Nacional Guanacaste, Estación Pitilla y Sendero El Mismo, 1059'26"N, 85²5'40"W, 700 m, 15 jun 1991, P. Ríos 365 (PT: USJ).

2. Dalechampia burgeriana Gómez-Laur., Novon 10(4): 368. 2000. Costa Rica: Puntarenas, Coto Brus, Fila Cruces, entrada de las Alturas y orilla de la quebrada, 1000-1200 m, 24 jul 1995, I. Chacón 279 (HT: USJ, IT: CR, F, MO).

\section{FABACEAE (s.1.)}

Caesalpinioideae

1. Copaifera camibar Poveda, N. Zamora \& P. Sánchez, Brenesia 31: 117. 1989 [1990]. Costa Rica: Puntarenas; Osa, Península de Osa, Alto de Mogos, 23 jul 1987, P. Sánchez \& L. Poveda 1272 (HT: CR, IT: B, F, K, LE, MEXU, MO, NY, P, QNCE, US, USJ, WIS).

2. Copaifera hemitomophylla Donn. Sm., Bot. Gaz. 27(5): 332. 1899. Costa Rica: Puntarenas; Golfito, Península de Osa, "Santo Domingo de Golfo Dulce" [área de Puerto Jiménez], mar 1896, A. Tonduz 9972 (HT: US, IT: CR, M, MO). USJ: Dos fotos y una fotocopia de dos isotipos ex M. Cynometra hemitomophylla (Donn. Sm.) Rose, N. Amer. Fl. 23(4): 220. 1930.

3. Sclerolobium costaricense N. Zamora \& Poveda, Novon 1(4): 199. 1991. Costa Rica: Alajuela; San Carlos, El Concho de Pocosol, $70 \mathrm{~m}$,

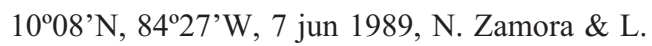
Poveda 1555 (HT: CR, IT: COL, F, MO, NY, PMA, US). Un isotipo en USJ.

Papilionoideae 
1. Phaseolus costaricensis G.F. Freytag \& D.G. Debouck, Novon 6(2): 157. 1996. Costa Rica: San José; San Isidro del General, 3 km SE de Copey, orillas del Río Pedregoso, 9³7'N, $83^{\circ} 55^{\prime} \mathrm{W}, 1800-2080 \mathrm{~m}, 14$ ene 1987, D.G. Debouck et al. 2135 (HT: US, IT: BR, CR, UC). Un isotipo en USJ.

2. Phaseolus talamancensis D.G. Debouck \& A.M. Torres Gonz., Novon 11(2): 280. 2001. Costa Rica: San José; San Isidro del General, 5 km N de Herradura, orillas del Río Blanco, al pie de la Fila Ojo de Agua, 9 $31^{\circ} \mathrm{N}, 83^{\circ} 37^{\prime} \mathrm{W}, 1890$ m, 13 ene 1987, D.G. Debouck 2130 (HT: CR, IT: COL, K, MO, US). Un isotipo en USJ.

\section{GESNERIACEAE}

1. Besleria imbricans Donn. Sm. var. uncinata C.V. Morton in Standl., Publ. Field Mus. Nat. Hist., Bot. Ser. 18(4): 1152. 1938. Costa Rica: Cartago; Tucurrique, Las Vueltas, 900-1000 m, A. Tonduz 13038 (HT: US). USJ: Foto y fotocopia de un isotipo ex M. Gasteranthus imbricans (Donn. Sm.) Wiehler, Selbyana 1(2): 155. 1975.

2. Drymonia submarginalis Gómez-Laur. \& M.M. Chavarría, Gesneriana 1(1): 15, f.1. 1995. Costa Rica: Alajuela; San Ramón, Los Ángeles, Reserva Biológica Alberto M. Brenes, sendero por la fila al NE de la estación, $10^{\circ} 13^{\prime} \mathrm{N}, 84^{\circ} 37^{\prime} \mathrm{W}$, 900-1050 m, 27 ago 1994 (fl), J. Gómez Laurito et al. 12675 (HT: USJ, IT: CR, F).

3. Paradrymonia bullata Gómez-Laur. \& M.M. Chavarría, Brenesia 33: 145, f.1. 1990 [1991]. Costa Rica: San José; Tarrazú, faldas del Cerro Nara, ca. Esquipulas y Villa Nueva, 9 30 ' $10^{\prime \prime} \mathrm{N}$, 8403'10"W, 300 m, 10 febr 1988, J. GómezLaurito 11686 (HT: CR; IT: F, USJ). La localidad es más detallada en la publicación.

\section{HAMAMELIDACEAE}

1. Distylium racemosum Sieb. \& Zucc., Fl. Jap. 1: 178, t.94. 1835. Japón; s.d., P. von Siebold s.n. (M). USJ: Foto del tipo ex M.

2. Molinadendron guatemalense (Radlk. ex Harms) Endress, Bot. Jahrb. Syst. 89(3): 357.
1969. Guatemala: Alta Verapaz; Cobán, 1350 m, blühend Jan. 1907, H. von Tuerckheim II 1613 (LT: M, ILT: A, B, BM, US). USJ: Foto del lectotipo ex M. Distylium guatemalense Radlk. ex Harms, Notizbl. Bot. Gart. Mus. Berlin-Dahlem 11(108): 716, Abb.13. 1933.

3. Sedgwickia cerasifolia Wall. \& Griff., As. Res. 19: 99, t.15. 1836. Malasia (Batavia); s.d., Herbar Griffith. USJ: Foto de un isotipo ex M. = Altingia excelsa Noronha, Verh. Batav. Genootsch. Kunsten 5(2): 9. 1790. = Liquidambar cerasifolia (Wall. \& Griff.) J.O. Voigt, Hort. Suburb. Calcutt.: 301.1845.

\section{HYDNORACEAE}

1. Prosopanche costaricensis L.D. Gómez \& Gómez-Laur., Phytologia 49(1): 53. 1981. Costa Rica: Limón; Siquirres, Finca La Lola, callejón sección 18, 50 m, 11 jun 1981. L.D. Gómez 7335 (HT: CR, IT: BM, F, MO, US, USJ).

\section{JUGLANDACEAE}

1. Alfaroa guanacastensis D.E. Stone, Fieldiana, Bot. 40: 40, f.7. 1977. Costa Rica: Guanacaste; southern slope of Volcán Tenorio, above upper pasture of Hacienda Tenorio, ca. $3700 \mathrm{ft}$. [1130 m], 21 Apr 1966, D.E. Stone 2167 (HT: DUKE; IT: A, CR, F, US). Un isotipo en USJ.

\section{LAMIACEAE}

1. Scutellaria tenuipetiolata A. Pool, Novon 8(1): 64. 1998. Costa Rica: Limón; Talamanca, Amubri, camino entre Amubri y Soki, siguiendo el Río Nabri hacia Alto Soki, 9²9'50"N, 8259'10"W, 150 m, 1 jul 1989 (fl, fr), G. Herrera 3101 (HT: MO, IT: CR, F). Un isotipo en USJ.

\section{LAURACEAE}

1. Licaria caribaea Gómez-Laur. \& Cascante, Novon 9(2): 199. 1999. Costa Rica: Limón; límite norte del Parque Nacional Tortuguero, Caño Suerte, 9 $9^{\circ} 36^{\prime} 42^{\prime \prime N}, 8^{\circ} 45^{\prime} 00^{\prime \prime} \mathrm{W}, 0-10 \mathrm{~m}, 2$ sept 1995 (fl, fru), A. Cascante \& A. Ruiz 705 (HT: 
CR, IT: F, MO, USJ).

2. Licaria leonis Gómez-Laur. \& A. Estrada, Lankesteriana 3: 5. 2002. Costa Rica: San José; Pérez Zeledón, Tinamaste, Finca Tinamaste $(17 \mathrm{~km}$ de San Isidro del General en camino a Dominical), bosque residual al pie de la Fila Tinamaste, $9^{\circ} 17^{\prime} 54^{\prime \prime} \mathrm{N}, 83^{\circ} 46^{\prime} 20^{\prime \prime} \mathrm{O}, 650-680 \mathrm{~m}, 10 \mathrm{mar}$ 1999 (fl), A. Estrada et al. 2085 (HT: CR, IT: F, MO, USJ).

3. Ocotea hartshorniana Hammel, J. Arnold Arbor. 67: 128. 1986. Costa Rica: Heredia; Finca La Selva, 2 May 1982, B. Hammel 11932 (HT: DUKE, IT: CAS, CR, F, MICH, MO, NY, US). Un isotipo en USJ.

4. Ocotea morae Gómez-Laur., Novon 7(2): 145. 1997. Costa Rica: Alajuela; San Ramón, Reserva Biológica Alberto Brenes, $10^{\circ} 13^{\prime} \mathrm{N}$, 84 ${ }^{\circ} 37^{\prime} \mathrm{W}, 800-850 \mathrm{~m}, 27$ ago 1995 (fl, fr), J. Gómez-Laurito \& V. Mora 12817 (HT: USJ, IT: CR, F, MO).

5. Ocotea pharomachrosorum Gómez-Laur., Novon 3(1): 31. 1993. Costa Rica: San José; Dota, San Gerardo, Finca de Efraín Chacón, $10^{\circ} 32^{\prime} 20^{\prime \prime} \mathrm{N}, 83^{\circ} 49^{\prime} 05^{\prime \prime} \mathrm{W}, 2100-2200 \mathrm{~m}, 10$ febr 1992, J. Gómez-Laurito et al. 12160 (HT: CR, IT: F, K, MO, USJ).

6. Pleurothyrium golfodulcense W. Burger \& N. Zamora, Fieldiana, Bot. n.s. 23: 115. 1990 ["golfodulcensis"]. Costa Rica: Puntarenas; Osa, Península de Osa, Alto de Mogos, camino a Rincón, 14 febr 1985, P.E. Sánchez et al. 1228 (HT: CR, IT: F, USJ).

7. Povedadaphne quadriporata $\mathrm{W}$. Burger, Brittonia 40(3): 277. 1988. Costa Rica: Alajuela; entre la Quebrada Palo y Ciudad Quesada, 27 jun 1983 (fl), L. Poveda et al. 3561 (HT: CR, IT: USJ). Dos isotipos en USJ. Ocotea quadriporata (W.C. Burger) Kosterm., Bot. Helv. 100(1): 36. 1990.

\section{LEPIDOBOTRYACEAE}

1. Ruptiliocarpon caracolito Hammel \& N. Zamora, Novon 3(4): 408. 1993. Costa Rica: Limon; Cordillera de Talamanca, cantón Matina, cuenca media del Río Barbilla, margen izquierda, sendero entre Cerro Amú, siguiendo la fila hacia el este, y estribaciones del Cerro Tigre, 959-60’ $\mathrm{N}$,
83²5-26’W, 200-700 m, 9 nov 1988 (fr), G. Herrera et al. 2310 (HT: CR, IT: AAU, BM, CAS, COL, DUKE, F, G, GB, K, KYO, LE, MEXU, MICH, MO, NY, PMA, QCA, QCNE, RSA, S, TEX, UC, US, USJ, VEN, WIS). Puntarenas; Osa, Sierpe, Península de Osa, Reserva Indígena Guaimí, 50-200 m, 20 oct 1990, B. Hammel et al. 17911 (PT: USJ). Loc. cit., Estación Agua Buena de Boscosa, 100-500 m, 28 nov 1990, B. Hammel 17983 (PT: USJ). Loc. cit., camino nuevo entre Rancho Quemado y Drake, 250 m, 20 mar 1991, B. Hammel et al. 18154 (PT: USJ, dos ejemplares). Loc. cit., Playa San Josecito, 1-10 m, 27 mar 1991, P. Harmon 210 (PT: USJ). Loc. cit., entre Chacarita y Rincón de Osa, 100 m, 27 mar 1991, R. Aguilar \& B. Hammel 101 (PT: USJ, dos ejemplares). Loc. cit., Fila Ganado hasta Guerra, 1-300 m, 28 mar 1991, B. Hammel et al. 18166 (PT: USJ).

\section{MALPIGHIACEAE}

1. Byrsonima herrerae W.R. Anderson, Contrib. Univ. Michigan Herb. 20: 22. 1995. Costa Rica: Limón; Talamanca, Alto Urén, subiendo a Cerro Chun siguiendo un antiguo camino de Quebrada Chaho a Alto Lari, 9²4'10"N, 8320'00"W, 800 m, 24 jul 1989 (fls.), G. Herrera 3333 (HT: MICH, IT: USJ). Limón; Talamanca, Bratsi, Alto Lari, siguiendo la fila entre Dapari y Río Lari, $9^{\circ} 25^{\prime} 50^{\prime \prime N}, 83^{\circ} 03^{\prime} 20^{\prime \prime} \mathrm{W}, 450$ m, 3 mar 1992, R. Aguilar \& H. Schmidt 1032 (PT: USJ).

\section{MELASTOMATACEAE}

1. Blakea costaricensis G. Umaña \& F. Almeda, Selbyana 12: 1. 1991. Costa Rica: Limón; Valle La Estrella, Fila Matama, El Progreso, $9^{\circ} 47^{\prime} 20^{\prime \prime} \mathrm{N}, 8^{\circ} 07^{\prime} 30^{\prime \prime} \mathrm{W}, 1600 \mathrm{~m}, 24$ abr 1989, G. Herrera \& A. Chacón 2758 (HT: CR, IT: CAS, COL, F, K, MEXU, MO, QCNE, US, USJ, WIS).

2. Blakea tapantiana G. Umaña \& F. Almeda, Novon 5(4): 305. 1995. Costa Rica: Cartago; Parque Nacional Tapantí, Sendero Oropéndola, $9^{\circ} 48^{\prime} 18^{\prime \prime} \mathrm{N}, 83^{\circ} 57^{\prime} 12^{\prime \prime} \mathrm{W}, 1200 \mathrm{~m}, 3$ sept 1992, G. Umaña et al. 497 (HT: CR, IT: CAS, COL, F, K, 
MEXU, MO, US, USJ, WIS). Un isotipo y un paratipo en USJ.

3. Miconia amplinodis G. Umaña \& F. Almeda, Novon 5(1): 110. 1995. Costa Rica: Alajuela; Bajos del Toro Amarillo, 10 $12^{\circ} 10^{\prime \prime N}$, $84^{\circ} 18^{\prime} 43^{\prime \prime W}, 1800$ m, 15 jun 1992, G. Umaña \& R. Chacón 495 (HT: CR, IT: CAS, COL, F, MEXU, MO, US, USJ). Loc. cit., 14 mayo 1992, G. Umaña et al. 484 (PT: USJ). Loc. cit., $10^{\circ} 13^{\prime} 00^{\prime \prime} \mathrm{N}, 8^{\circ} 18^{\prime} 00^{\prime \prime} \mathrm{W}, 6$ ene 1994, G. Umaña et al. 510 (PT: USJ).

4. Miconia friedmaniorum F. Almeda \& G. Umaña, Novon 3(1): 5. 1993. Costa Rica: Alajuela; Upala, Colonia Libertad, subiendo hasta el Llano Aguacatales, $10^{\circ} 48^{\prime} 25^{\prime \prime} \mathrm{N}$, $85^{\circ} 17^{\prime} 50^{\prime \prime W}, 1500$ m, 28 abr 1988, G. Herrera 1900 (HT: CR, IT: CAS, F, MEXU, MO, PMA, USJ). Alajuela; San Ramón, Ángeles Norte, camino de entrada a la Reserva Biológica A.M. Brenes, cerca de la Estación, $10^{\circ} 13^{\prime} \mathrm{N}$, 84³7'W, 900 m, 4 mayo 1991, J. Gómez Laurito \& V. Mora 12073 (PT: USJ).

5. Miconia pendula G. Umaña \& F. Almeda, Novon 3(1): 7, f.2. 1993. Costa Rica: Cartago; Refugio Nacional de Vida Silvestre Tapantí, orilla de Sendero Los Palmitos, 09 44'00"N, 8347'00"W, 1300-1400 m, 2 ago 1990, G. Umaña et al. 391 (HT: CR; IT: BM, BR, CAS, COL, CR, F, MEXU, MO, NY, PMA, US, USJ).

6. Triolena pumila G. Umaña \& F. Almeda, Brittonia 43(3): 149. 1991. Costa Rica: Limón; Cordillera de Talamanca, Matina, Barbilla, en confluencia de Río Dantas y Río Cañón Seco (Quebrada Barreal), $10^{\circ} 00^{\prime} 30^{\prime \prime} \mathrm{N}, 83^{\circ} 25^{\prime} 00^{\prime \prime} \mathrm{W}, 200$ 300 m, 18 oct 1988, G. Herrera 2209 (HT: CR, IT: CAS, MO, USJ).

\section{MONIMIACEAE}

1. Mollinedia selloi A. DC., Prod. 16 (2): 666 1868. Brasil, s.d., Sello 595 (13461?) (B). En USJ, copia de una foto ex F.

\section{MORACEAE}

1. Trophis macrostachya Donn. Sm., Bot. Gaz. 40(1): 10. 1905. Costa Rica: Cartago; Jiménez, Tu- currique, pascuis ad Las Vueltas, nov 1898, A. Tonduz 8124 (CR-12802) (LT: US, designado por W. Burger, Lanj. \& Wess. Boer, Acta Bot. Neerl. 11: 449. 1962). USJ: Foto más fotocopia del ILT A. Tonduz 12802 ex M. = Sorocea pubivena Hemsl., Biol. Centrali-Amer., Bot. 3(15): 150. 1883

\section{MYRTACEAE}

1. Marlierea mesoamericana P.E. Sánchez, Brenesia 35: 117. 1991. Costa Rica: Heredia; Parque Nacional Braulio Carrillo, carretera entre San José y Guápiles, Estación La Montura, $10^{\circ} 07^{\prime} 00^{\prime \prime N}, 83^{\circ} 58^{\prime} 30^{\prime \prime} \mathrm{W}, 800 \mathrm{~m}, 9$ jul 1989, P. Sánchez et al. 1305 (HT: CR, IT: BM, F, K, ME$\mathrm{XU}, \mathrm{MO}, \mathrm{NY}, \mathrm{US}, \mathrm{WIS})$. Un isotipo en USJ.

2. Plinia cuspidata Gómez-Laur. \& Valverde, Lankesteriana 3: 11. 2002. Costa Rica: Limón; Talamanca, Bratsi, Buena Vista, Finca ACODEFO, lomas de Fila Carbón, 9³9’30"N, 8352’30"W, 300 m, 25 nov 2000, O. Valverde \& S. Mora 1339 (HT: USJ). Algunos detalles de fecha y localidad del ejemplar tipo difieren de la publicación; sin embargo, Valverde \& Mora 1339 es sin duda el holotipo.

3. Plinia puriscalensis P. Sánchez \& Q. Jiménez, Brenesia 32: 113. 1989 [1990]. Costa Rica: San José; Puriscal, Santa Rosa, Zona Protectora La Cangreja, 9०42'20"N, 84'23'35"W, 400 m, 29 abr 1988 (fl), Q. Jiménez \& P.E. Sánchez 586 (HT: CR, IT: F, K, MO). Un isotipo en USJ.

\section{NYCTAGINACEAE}

1. Neea popenoei P.H. Allen, Rain For. Golfo Dulce: 273 , 410. 1956. Costa Rica; Osa, margins of forest near Palmar Norte, $50 \mathrm{ft}$. [15 m], 31 Mar 1949, P.H. Allen 5225 (HT: EAP). USJ: Foto más fotocopia de un isotipo ex $\mathrm{M}$.

\section{PASSIFLORACEAE}

1. Passiflora boenderi J.M. MacDougal, Novon 13(4): 454, f.1-2. 2003. Costa Rica: Heredia; Sarapiquí, Cariblanco, camino a Virgen del Socorro, ca. 750 m, 8 oct 1992, A. Vega s.n. (PT: USJ-50082). 
2. Passiflora tica Gómez-Laur. \& L.D. Gómez, Phytologia 49(1): 56. 1981. Costa Rica: Alajuela; San Ramón, a la vera del río San Lorenzo, faldas de la Fila Volcán Muerto, 1100 m, 4 ago 1979, J. Gómez Laurito 5019 (HT: CR). Un ejemplar en USJ anotado como isotipo. El número de recolecta citado en TRÓPICOS es diferente (Gómez Laurito 6627). Cartago; Turrialba, entre Chitaría y Tres Equis, 22 abr 1975, L. Poveda 967 (PT: USJ).

\section{POLYGONACEAE}

1. Coccoloba bejuco Poveda \& P.E. Sánchez, Brenesia 34: 163. 1990 [1991]. Costa Rica: Puntarenas; Osa, Península de Osa, Alto de los Mogos, del Rancho Maracaná 3 km camino a Rincón, sendero a Punta Pargo, 27 oct 1989, L. Poveda \& P. Sánchez 4649 (HT: CR, IT: F, K, MEXU, MO). Un isotipo en USJ.

2. Coccoloba porphyrostachys Gómez-Laur., Brenesia 31: 121. 1989 [1990]. Costa Rica: Alajuela; Reserva Forestal de San Ramón, Estación Río San Lorencito, a la orilla del camino, $10^{\circ} 13^{\prime} \mathrm{N}$, $84^{\circ} 36^{\prime} \mathrm{W}, 850 \mathrm{~m}, 2$ oct 1989, R. Ortiz \& V. Mora 310 (HT: CR, IT: F, MO, USJ).

\section{RUBIACEAE}

1. Coussarea loftonii (Dwyer \& M.V Hayden) Dwyer subsp. occidentalis C.M. Taylor, Novon 11(1): 140, f.1. 2001. Costa Rica: Puntarenas; Osa, Península de Osa, Parque Nacional Corcovado, Los Planes, La Gloria, 8 37'30"N, 8340'50"W, 170 m, 16 febr 1991, G. Induni 255 (HT: INB, IT: MO). Un isotipo en USJ. Faramea loftonii Dwyer \& M.V. Hayden, Phytologia 15(1): 56.1967.

2. Isertia scorpioides B.M. Boom, Brittonia 36(4): 435. 1984. Panamá: Darién; "Penas Bay near hotel", 20 Jun 1969, E.L. Tyson 5537 (PT: USJ).

3. Psychotria grandis Sw., Prodr.: 43. 1788. Jamaica interioris occidentalis, s.d., O. Swartz s.n. (HT: S). USJ: Foto de un isotipo ex M.

4. Rondeletia povedae Lorence, Fieldiana, Bot. n.s. 33: 299, f.41A. 1993. Costa Rica: San José; Puriscal, Santa Rosa, 17 ene 1973, L. Poveda 441
(HT: CR, IT: MO). Un isotipo en USJ. Arachnothryx povedae (Lorence) Borhidi, Acta Bot. Hung. 38(1-4): 140. 1993-1994 [1995].

5. Rudgea monofructus Gómez-Laur. \& Dwyer, Novon 1(1): 50. 1991. Costa Rica: Alajuela; Reserva Forestal de San Ramón, campamento Río San Lorencito, $10^{\circ} 13^{\prime} \mathrm{N}, 84^{\circ} 37^{\prime} \mathrm{W}, 900-1100 \mathrm{~m}, 2$ sept 1989, J. Gómez-Laurito \& G. Vargas 11822 (HT: CR, IT: F, MO, USJ). Dos isotipos en USJ: uno seco y otro en líquido.

6. Rudgea trifurcata Gómez-Laur., Brenesia 33: 139. 1990. Costa Rica: Limón; Talamanca, 3 km al oeste de Volio, 09 $37^{\prime} 40^{\prime \prime} \mathrm{N}, 82^{\circ} 533^{\prime} 50^{\prime \prime} \mathrm{W}, 100$ m, 6 mayo 1990, J. Gómez-Laurito \& G. Vargas 11957 (HT: CR, IT: F, MO, USJ).

7. Schradera reticulata J. Sánchez-Gonz., Novon 13(4): 477. 2003. Costa Rica: Limón; Talamanca, Sixaola, Gandoca, El Llano entre filas Manzanillo y Río Mile Creek, 9³7'00"N, 8241'00"W, 50-100 m, 27 mar 1995, G. Herrera \& E. Sandoval 7585 (HT: CR, IT: F, K, MO, US, USJ). "Braulio Carrillo" (sin más datos), 8 abr 1983, G. Stiles 83-70 (PT: USJ).

\section{RUTACEAE}

1. Amyris magnifolia Gómez-Laur. \& Q. Jiménez, Lankesteriana 6: 5. 2003. Costa Rica: Puntarenas; Osa, Península de Osa, Reserva Forestal Golfo Dulce, Rancho Quemado, Fila al oeste de Tierra de Conservación, camino a Cerro Brujo, $8^{\circ} 46^{\prime} 20^{\prime \prime} \mathrm{N}, 83^{\circ} 22^{\prime} 40^{\prime \prime O}, 200 \mathrm{~m}, 15$ nov 1993 (fl, fr), R. Aguilar \& B. Hammel 2620 (HT: INB, IT: MO, NY, USJ). Puntarenas; Golfito, Valle de Coto Colorado, Refugio Nacional de Vida Silvestre Golfito, $3 \mathrm{~km}$ al $\mathrm{N}$ del aeropuerto, $8^{\circ} 40^{\prime} 25^{\prime \prime} \mathrm{N}, 83^{\circ} 11^{\prime} 20^{\prime \prime} \mathrm{W}, 200 \mathrm{~m}, 27$ ene 1992 (fr), C. Formoso et al. 8 (PT: USJ). Puntarenas; Osa, Península de Osa, Los Mogos, Bahía Chal, $8^{\circ} 46^{\prime} 20^{\prime \prime} \mathrm{N}, 8^{\circ} 22^{\prime} 40^{\prime \prime} \mathrm{W}, 200 \mathrm{~m}, 23$ ago 1994, R. Aguilar et al. 3579 (PT: USJ). Puntarenas; Osa ("Golfito"), Sierpe, Península de Osa, Estero Guerra, $8^{\circ} 46^{\prime} 00^{\prime \prime} \mathrm{N}, 83^{\circ} 35^{\prime} 10^{\prime \prime} \mathrm{W}, 200 \mathrm{~m}, 6$ jun 1995, A. Estrada 425 (PT: USJ). Puntarenas; Golfito, entre Golfito y La Gamba, sendero a Cerro Adams [hacia las torres de comunicación], 840’40"N, 83¹1'55"W, 160-190 m, 26 
oct 2002 (fr), J. Gómez Laurito et al. 13910 (PT: USJ).

2. Galipea dasysperma Gómez-Laur. \& Q. Jiménez, Novon 4(4): 347. 1994. Costa Rica: Puntarenas; Osa, Sierpe, Península de Osa, camino entre Rancho Quemado y Sierpe, 8 $44^{\prime} 10^{\prime \prime} \mathrm{N}$, $83^{\circ} 35^{\prime} 30^{\prime \prime W}, 100$ m, 21 jun 1990, G. Herrera 4228 (HT: CR, IT: F, K, MO, NY, USJ).

3. Peltostigma parviflorum Q. Jiménez \& Gereau, Ann. Missouri Bot. Gard. 78(2): 527, f.1. 1991. Costa Rica: San José; Puriscal, Santa Rosa, Zona Protectora La Cangreja, 943'10"N, 84²3'10"W, 400-500 m, 4 sept 1987 (fl), Q. Jiménez \& L. Poveda 494 (HT: CR, IT: F, K, MO). Un isotipo en USJ. = Peltostigma guatemalense (Standl. \& Steyerm.) Gereau, Novon 5(1): 34. 1995. Galipea guatemalensis Standl. \& Steyerm., Publ. Field Mus. Nat. Hist., Bot. Ser. 23(4): 165. 1944.

\section{SYMPLOCACEAE}

1. Symplocos naniflora L. Kelly \& F. Almeda, Novon 12(3): 374, f.3. 2002. Costa Rica: Puntarenas; Golfito, Jiménez, Península de Osa, entre Quebrada Patemazo y las cabeceras del Río Madrigal, $8^{\circ} 30^{\prime} 15^{\prime \prime} \mathrm{N}, 83^{\circ} 28^{\prime} 50^{\prime \prime} \mathrm{W}, 650 \mathrm{~m}, 30$ nov 1990, G. Herrera 4677 (HT: CAS, IT: CR, F, MEXU, MO, NY). Un isotipo en USJ.

2. Symplocos povedae Almeda, Bull. Torrey Bot. Club 109(3): 320, f.2. 1982. Costa Rica: Heredia; Monte de la Cruz, entre las piedras a la vera del Río Patria, 30 oct 1975, L. Poveda 1179 (HT: CAS, IT: CR, F, NY). Un isotipo en USJ.

\section{THEACEAE}

1. Ternstroemia multiovulata Gómez-Laur., Q. Jiménez \& N. Zamora, Brenesia 33: 127, f.1. 1990 [1991] ["multiovat","multiovata"]. Costa Rica: Puntarenas; Osa, Península de Osa, Parque Nacional Corcovado, Llorona to Los Planes, $8^{\circ} 27-30^{\prime} \mathrm{N}, 83^{\circ} 33-38^{\prime} \mathrm{W}, 100$ m, 25 Mar 1989, C. Kernan \& P. Phillips 1006 (HT: CR, IT: F, MO, USJ).

\section{THEOPHRASTACEAE}

1. Deherainia lageniformis Gómez-Laur. \& N. Zamora, Novon 8(2): 141. 1998. Costa Rica: Guanacaste; Cañas, Parcelas Nueva Guatemala, Estribaciones del Volcán Tenorio, $1000 \mathrm{~m}$, $10^{\circ} 37^{\prime} 30^{\prime \prime} \mathrm{N}, 85^{\circ} 00^{\prime} 45^{\prime \prime} \mathrm{W}, 18$ ene 1994, G. Herrera 6779 (HT: USJ, IT: CR, F, INB, MO).

\section{TICODENDRACEAE}

1. Ticodendron incognitum Gómez-Laur. \& L.D. Gómez, Ann. Missouri Bot. Gard. 76: 1148. 1989. Costa Rica: Puntarenas ["Alajuela"]; Reserva Biológica Monteverde, Río Peñas Blancas, $10^{\circ} 20^{\prime} \mathrm{N}, 84^{\circ} 43^{\prime} \mathrm{W}, 850-900 \mathrm{~m}, 28$ mar 1987, W. Haber \& E. Bello 6840 (PT: USJ). San José; Moravia, Bajo de la Hondura, 21 mayo 1974, L. Poveda 864 (PT: USJ).

\section{VERBENACEAE}

1. Citharexylum costaricense Moldenke, Repert. Spec. Nov. Regni Veg. 37(14): 219. 1934. Costa Rica: San José; La Palma, 1460 m, 19 sept 1898, A. Tonduz 12557 (HT: F). USJ: Foto de un isotipo ex M.

\section{Clase LILIOPSIDA (= Monocotyledoneae)}

\section{ALISMATACEAE}

1. Echinodorus botanicorum L.D. Gómez \& Gómez-Laur., Phytologia 51(7): 476. 1982. Costa Rica: Puntarenas; Buenos Aires ("Buenos Aires de Osa"), 10 abr 1977, M. Bermúdez 741 (PT: USJ). = Sagittaria rhombifolia Cham., Linnaea 10: 219. 1835.

\section{ARACEAE}

1. Stenospermation pteropus Grayum, Phytologia 82(1): 52. 1997. Costa Rica: Limón; Alto Urén, subiendo por la fila entre la margen derecha de la Quebrada Chaho y la margen izquierda del Río Lorni, Cerro Láubeta, 9 ${ }^{\circ} 23^{\prime} 10^{\prime \prime} \mathrm{N}, 83^{\circ} 00^{\prime} 25^{\prime \prime} \mathrm{W}$, 1190 m, 26 jul 1989, G. Herrera 3353 (HT: MO, IT: CR, K, US). Un isotipo en USJ. 
2. Syngonium castroi Grayum, Phytologia 82(1): 52. 1997. Costa Rica: Puntarenas; Osa, Península de Osa, Parque Nacional Corcovado, Cerro Brujo, $8^{\circ} 38^{\prime} \mathrm{N}, 83^{\circ} 35^{\prime} \mathrm{W}, 600 \mathrm{~m}, 23$ ene 1991, E. Castro 242 (HT: USJ, unicatum!).

3. Xanthosoma croatianum L.D. Gómez \& Gómez-Laur., Phytologia 52: 227. 1982 ["croatana"]. Costa Rica: Guanacaste; Abangares, Pozo Azul, 29 ago 1981, C.E. Valerio s.n. (PT: USJ23464 y 23465). = Xanthosoma mexicanum Liebm., Vidensk. Meddel. Dansk Naturhist. Foren. Kjobenhavn: 15. 1849.

\section{ARECACEAE}

1. Bactris ana-juliae Cascante, Palms 44(3): 146. 2000. Costa Rica: San José; Pérez Zeledón, Fila Tinamaste, $17 \mathrm{~km}$ de San Isidro a Dominical, $1000 \mathrm{~m}, 9^{\circ} 17^{\prime} 40^{\prime \prime} \mathrm{N}, 8^{\circ} 46^{\prime} 00^{\prime \prime} \mathrm{W}, 28$ mayo 1998 , O. Valverde 993 (HT: CR, IT: MO, USJ).

2. Bactris herrerana Cascante, Palms 44(3): 148, f.2. 2000. Costa Rica: San José; Pérez Zeledón, San Cristóbal, Finca Tinamaste, 17 km de San Isidro a Dominical, al pie de Fila Tinamaste, 650-680 m, 9¹7'54"N, 83ํ6'20"W, 2 dic 1998, A. Cascante et al. 1470 (HT: CR, IT: MO, USJ).

3. Chamaedorea matae Hodel, Principes 35: 75. 1991. Costa Rica: Puntarenas; Osa, Península de Osa, Rincón, 1 febr 1964, G. Mata 497 (HT: CR). Un isotipo en USJ. Henderson et al. (1995) tratan este nombre como sinónimo de C. warscewiczii H. Wendl., no así Grayum (2003).

4. Chamaedorea piscifolia Hodel, G. Herrera \& Cascante, Palm J. 137: 32, f.1-2. 1997. Costa Rica: San José; Tarrazú, $6 \mathrm{~km} \mathrm{~S}$ of Nápoles, Chilamate, west side of Cerro Pito, 1200 m, 5 Dec 1996, D.R. Hodel et al. 1540 (HT: BH, IT: CR, K, MO, NY, USJ).

5. Chamaedorea rossteniorum Hodel, G. Herrera \& Cascante, Palm J. 137: 34, f.3-6. 1997. Costa Rica: San José; Tarrazú, 7 km S of San Lorenzo, Cruce de Pacaya, 1600 m, 4 Dec 1996, D.R. Hodel et al. 1525 (HT: BH, IT: CR, MO, USJ). Antes de la publicación, ejemplares anotados como "Chamaedorea bakeri Herrera, Cascante \& Hodel", nom. herb.

\section{CYPERACEAE}

1. Cyperus turrialbanus Gómez-Laur., Brenesia 14-15: 357, f.1. 1978. Costa Rica: Cartago; Turrialba, $9^{\circ} 54^{\prime} \mathrm{N}, 83^{\circ} 38^{\prime} \mathrm{W}$, "in regime humidissima silvae premontana, apud Reventazonis fluminis ripam circa $1000 \mathrm{~m}$ adversus flumen, in confluentia fluvii Turrialbae lecta, elevatio loci circa 600 m", febr 1977, J. Gómez Laurito 2331 (HT: CR, IT: USJ).

2. Mapania herrerae Gómez-Laur., Phytologia 60(1): 73. 1986. Costa Rica: Limón; camino de Fila Dimat-Soki hacia Soki, Quebrada Sha, $9^{\circ} 29^{\prime} \mathrm{N}, 82^{\circ} 58^{\prime} \mathrm{W}, 150 \mathrm{~m}, 26$ oct 1985, L.D. Gómez et al. 23860 (HT: CR, IT: F, MO, USJ). = Mapania cuatrecasasii T. Koyama, Mem. New York Bot. Gard. 17(1): 59, f.12. 1967.

3. Rhynchospora cabecarae Gómez-Laur., Novon 5(3): 270. 1995. Costa Rica: Limón; Talamanca; $\mathrm{N}$ flank of Cerro Casma, along Ujarrás-San José Cabécar trail, Cordillera de Talamanca, $9^{\circ} 20^{\prime} 30^{\prime \prime} \mathrm{N}, 8^{\circ} 13^{\prime} 30^{\prime \prime} \mathrm{W}, 2250-2270 \mathrm{~m}, 17$ Mar 1993, M. Grayum 10354 (HT: USJ).

4. Rhynchospora carrillensis Gómez-Laur., Brenesia 22: 353. 1984. Costa Rica: San José; Parque Nacional Braulio Carrillo, La Montura, $10^{\circ} 03^{\prime} \mathrm{N}$, 8402'W, 1100 m, 25-30 jul 1982, C. Todzia et al. 1994, (HT: CR, IT: NY). Un isotipo en USJ.

5. Uncinia koyamae Gómez-Laur., Brenesia 18: 92. 1980 [“koyamai”]. Costa Rica: Limón; Macizo del Chirripó [vertiente atlántica], ca. $150 \mathrm{~m}$ al $\mathrm{N}$ del Lago Ditkebi, $9^{\circ} 28^{\prime} \mathrm{N}, 83^{\circ} 28^{\prime} \mathrm{W}, 3600 \mathrm{~m}$, 18 mar 1979, J. Gómez Laurito 4770 (HT: CR, IT: F). Dos isotipos en USJ.

\section{HELICONIACEAE}

1. Heliconia rodriguezii F.G. Stiles, Brenesia 1920: 222. 1982. Costa Rica: San José; Parque Nacional Braulio Carrillo, La Montura, 1050 m, 27 Jul 1980 (HT: USJ, IT: CR, F, M, ULM, US, USJ). En la publicación, los acrónimos son incorrectos: "UCR (holotype), MNCR, FMNH, USNM". Además, los isotipos fueron distribuidos en mayo y junio de 2002 (vide Morales 2002).

2. Heliconia vulcanicola F.G. Stiles, Brenesia 18: 
148. 1980. Costa Rica: Guanacaste; Volcán Orosi, $10^{\circ} 58^{\prime} \mathrm{N}, 85^{\circ} 28^{\prime} \mathrm{W}, 1360 \mathrm{~m}$, mayo 1979 , F.G. Stiles 79-1 (HT: USJ, IT: CR, F, US). Como en el caso anterior, los acrónimos citados en la publicación son erróneos: "UCR" es USJ, "MNCR" es CR, "FMNH" es F y "USNM" es US. = Heliconia monteverdensis G.S. Daniels \& F.G. Stiles var. vulcanicola (F.G. Stiles) W.J. Kress, Selbyana 11: 53. 1989.

\section{ORCHIDACEAE}

1. Chondrorhyncha lankesteriana Pupulin, Lindleyana 15(1): 21. 2000. Costa Rica; without definite locality, a confisticated plant flowered in cultivation at Jardín Botánico Lankester at Paraíso de Cartago, 1400 m, 19 May 1999, F. Pupulin 1467 (HT: USJ, IT: SEL en líquido). Además, un clonotipo en USJ: F. Pupulin 2255.

2. Chondroscaphe yamilethae Pupulin, ined., Costa Rica: Puntarenas; Buenos Aires, Holán, 12001300 m, collected by C. Arguedas, 2000, flowered in cultivation in the collection of J. Cambronero in San Isidro de Pérez Zeledón, $20 \mathrm{Apr}$ 2003, F. Pupulin 4701 (HT: USJ, con una flor en líquido).

3. Coryanthes kaiseriana G. Gerlach, Lankesteriana 8: 23. 2003. Costa Rica: San José; Pérez Zeledón, San Isidro, cult. Danilo Quesada Rivera (VI Exposición Nacional de Orquídeas), testigo para dibujo \# 918, 27 mar 1976, R.L. Rodríguez 1497 (HT: USJ). La ilustración que preparó R.L. Rodríguez permanece inédita [como Coryanthes speciosa (Hook.) Hook.].

4. Crossoglossa aurantilineata Pupulin, Lindleyana 15(1): 23. 2000. Costa Rica: Cartago; Turrialba, road between Torito and Bonilla Arriba, 1480 m, 4 Aug 1998, flowered in cultivation at Jardín Botánico Lankester at Paraíso de Cartago, 15 May 1999, F. Pupulin et al. 990 (HT: USJ, IT: SEL en líquido).

5. Dichaea filiarum Pupulin, ined., Costa Rica: Cartago; Turrialba, Monumento Nacional Guayabo, $9^{\circ} 56^{\prime} \mathrm{N}, 83^{\circ} 43^{\prime} \mathrm{W}$, ca. $800 \mathrm{~m}, 9$ Aug 2003 , flowered in cultivation at Jardín Botánico Lankester, 8 Oct 2003, F. Pupulin et al. 4944 (HT:
USJ). En USJ, además, una lámina con ilustraciones y una foto en colores de la planta in vivo.

6. Dracula inexperata Pupulin, Orchids 70(6): 564. 2001. Costa Rica: Cartago; Paraíso, Orosi, Parque Nacional Tapantí, sendero Oropéndola, along the Río Grande de Orosi, 1160 m, 19 Nov 2000, F. Pupulin \& R. Chacón 2584 (HT: USJ, unicatum!).

7. Encyclia ortizii Dressler, Novon 5(2): 140. 1995. Costa Rica: Alajuela; Ángeles Norte, Reserva Forestal de San Ramón, aprox. 30 km NNO de San Ramón, 8-9 dic 1984, R.L. Dressler \& Biología-350 \# 288 (HT: USJ). = Prosthechea ortizii (Dressler) W.E. Higgins, Phytologia 82(5): 379. 1997.

8. Epidendrum ingramii Hágsater \& García-Cruz, Icon. Orchid. 3(2): t.338. 1999. Costa Rica: Puntarenas; Monteverde Reserve, $2 \mathrm{~km}$ SW Station, $10^{\circ} 18^{\prime} \mathrm{N}, 84^{\circ} 48^{\prime} \mathrm{W}, 1500-1550 \mathrm{~m}, 30 \mathrm{Jul} 1992, S$. Ingram \& K. Ferrell 1593 (HT: AMO). Un isotipo en USJ.

9. Epidendrum monophlebium Hágsater, Icon. Orch. 3: t.358. 1999. Costa Rica: Cartago; vertiente atlántica oriental, orillas del Río Purisil, 1350 m, 20 nov 1982, C. Horich sub E. Hágsater 6932 (HT: USJ, IT: AMO (ilustr.), SEL). En USJ, además, una lámina con copias de la publicación y las ilustraciones originales.

10. Epidendrum montis-narae Pupulin \& L. Sánchez, Lankesteriana 1: 7. 2001. Costa Rica: San José; Dota, eastern slopes and crest of Cerro Nara, 1050-1140 m, 15 Jan 1999, F. Pupulin et al. 1140 (HT: USJ, CT: AMO). Costa Rica; without specific locality, a confiscated plant flowered at Gaia Botanical Garden, 28 Apr 1999, GBG-1353 (PT: USJ).

11. Fernandezia tica Mora-Ret. \& J. García, Brenesia 39-40: 164. 1993. Costa Rica: Heredia; carretera a Vara Blanca, 2000 m, 27 oct 1992, J.T. Atwood \& O. Rodríguez 4181 (HT: USJ, unicatum!).

12. Govenia viaria Dressler, Lankesteriana 3: 26, f.1. 2002. Costa Rica: San José; Panamerican Highway south of Cartago, about km. 46, 21 Jul 1994, R.L. Dressler \& D.E. Mora 6168 (HT: MO, IT: USJ). En USJ hay dos isotipos, que en 
el pasado fueron parcialmente dañados por insectos.

13. Ionopsis papillosa Pupulin, Harvard Pap. Bot. 3: 227, f.2. 1998. Ecuador: Pichincha; Santo Domingo de los Colorados, ca. $500 \mathrm{~m}$, collected by A. Olmi, Sept 1995, flowered in cultivation, 1 May 1997, F. Pupulin 402 (HT: SEL). Un isotipo en USJ.

14. Kefersteinia alata Pupulin, Harvard Pap. Bot. 8(2): 161. 2004. Panamá: Bocas del Toro; road from David to Chiriquí Grande, around km 74, ca. 450 m, 10 Apr 2001, flowered in cultivation at Gaia Botanical Garden (Quepos, Costa Rica), 18 Aug 2001, F. Pupulin et al. 3119 (HT: USJ, IT: USJ en líquido).

15. Kefersteinia excentrica Dressler \& Mora-Ret., Orquídea (Méx.) 13: 261. 1993. Costa Rica: Cartago, La Selva, camino a Taus, en la misma ruta al Refugio de Fauna Silvestre Tapantí, 13001400 m, 9 nov 1984, floreció en febr 1991, R.L. Dressler \& D.E. Mora s.n. (HT: USJ-45426, IT: FLAS, USJ en líquido). Alajuela; San Ramón, Cataratas, jul 1990, D.E. Mora s.n. (PT: USJ45427, con un duplicado en líquido).

16. Kefersteinia orbicularis Pupulin, Lindleyana 15(1): 25, f.3. 2000. Costa Rica: San José; Dota, crest of Cerro Nara, 1100 m, 15 Jan 1999, F. Pupulin et al. 1170 (HT: USJ, IT: SEL en líquido). Misma localidad y fecha, F. Pupulin et al. 1127 (PT: USJ).

17. Kefersteinia retanae Gerlach ex C.O. Morales, Brenesia 52: 75. 1999 [2000]. Costa Rica: San José; Pérez Zeledón, Peña Blanca, 600 m, floreció en cultivo en el Jardín Botánico de Múnich (Alemania), jun 1992, G. Gerlach s.n. (HT: USJ-69249, IT: M, Herb. Königer). Vide Gerlach (1994).

18. Lepanthes casasae Pupulin, Orchidee (Hamburg) 54(4): 472. 2003. Costa Rica: Alajuela; Reserva Monteverde, collected by estudiantes de biología, 6 Jul 1997, flowered in cultivation at Jardín Botánico Lankester, 21 May 2002, F. Pupulin 3855 (HT: USJ en líquido, IT: USJ).

19. Lepanthes gerardensis M.A. Blanco, Lankesteriana 8: 19. 2003. Costa Rica: Puntarenas; Monteverde, Valle de San Gerardo, entre Santa Elena y Arenal, vertiente atlántica, ca. $10^{\circ} 23^{\prime} 00^{\prime \prime} \mathrm{N}$,
8447'50"O, 1000 m, 23 ene 1999, M. Blanco \& V. Arias 924 (HT: USJ).

20. Lepanthes ingramii Luer, Lindleyana 7(2): 108, f.7. 1992. Costa Rica: San José; Vásquez de Coronado, Parque Nacional Braulio Carrillo, along Sendero Botella, 750 m, 21 Sept 1990, S. Ingram \& K. Ferrell 561 (HT: SEL, IT: MO). Un isotipo fue robado de USJ ca. 1995.

21. Lepanthes johnsonii Ames ssp. costaricensis $\mathrm{Pu}-$ pulin, Harvard Pap. Bot. 6(1): 291. 2001. Costa Rica: Cartago; Orosi, road to the Parque Nacional Tapantí, about $1 \mathrm{~km}$ before the entrance of the Park, 1160 m, 6 Febr 2000, F. Pupulin 1979 (HT: USJ). Misma localidad, 1170 m, 7 May 2000, F. Pupulin \& Curso de Orquideología 2409 (PT: USJ). Todo el material original se perdió antes de llegar a USJ; por tanto, será necesario designar un neotipo.

22. Lepanthes rafaeliana Pupulin, Harvard Pap. Bot. 6(1): 289. 2001. Costa Rica: Cartago ["San José"]; Guarco, San Isidro, Ojo de Agua, km 76-77, Carretera Interamericana sur, 2800-2900 m, 14 abr 1967, H. Hespenheide s.n. (HT: USJ-10846, con copia del dibujo \# 494 de R.L. Rodríguez).

23. Lepanthes schugii Pupulin, Orchidee (Hamburg) 54(4): 474. 2003. Costa Rica: San José; Dota, Santa María, Las Quebradillas, 0941'32"N, 8358'30"W, 2100 m, 22 abr 2001, W. Schug s.n. (HT: USJ-81484, en líquido).

24. Lepanthes spadariae Pupulin, Harvard Pap. Bot. 6(1): 291. 2001 ["spadarii"]. Costa Rica: San José; Pérez Zeledón, San Ramón Norte, trail to Cerro Pelón, near the summit of the mountain, 1640 m, 3 May 2000, F. Pupulin et al. 2372 (HT: USJ, IT: SEL en líquido).

25. Lepanthes whittenii Pupulin \& Bogarín, ined. Costa Rica: Cartago; El Guarco, El Empalme, $\mathrm{km} \mathrm{52,} \mathrm{Carretera} \mathrm{Interamericana} \mathrm{sur,}$ $9^{\circ} 42^{\prime} 49.1^{\prime \prime N}, 83^{\circ} 56^{\prime} 58.2^{\prime \prime} \mathrm{W}, 2295$ m, 23 July 2003, M. Whitten et al. 2164 (HT: USJ).

26. Lockhartia pandurata Pupulin, Rev. Biol. Trop. 46(4): 998. 1998. Costa Rica: Puntarenas; Aguirre, Quepos, Parque Nacional Manuel Antonio, 85 m, 11 jul 1995, floreció en cultivo, 4 mayo 1996, F. Pupulin 313 (HT: USJ, IT: CR). También un IT en USJ. = Lockhartia micrantha Rchb.f., Bot. Zeit. 10: 767. 1852. 
27. Macroclinium confertum Pupulin, Lindleyana 11(3): 138, f.2. 1996. Costa Rica: San José; Dota, San Marcos, road to San Joaquín, 1455 m, 28 Apr 1992, flowered in cultivation, $4 \mathrm{Jul}$ 1992, $F$. Pupulin 204 (HT: USJ).

28. Macroclinium doderoi Mora-Ret. \& Pupulin, Selbyana 18(1): 7. 1997. Costa Rica: Cartago; Turrialba, Caño Seco, Torito, a lo largo de un tributario menor del Río Torito, ca. 1500 m, 19 jul 1994, R.L. Dressler et al. s.n. (HT: USJ-57025, IT: USJ en líquido). Cartago; Turrialba, Guayabo, near La Fuente, F. Pupulin et al. 234 (PT: USJ).

29. Macroclinium generalense Pupulin, Lindleyana 11(3): 136, f.1. 1996. Costa Rica: San José; Pérez Zeledón, Alto de San Juan, along the road to Dominical, 1090 m, Mar 1989, F. Pupulin 24 (HT: USJ).

30. Macroclinium robustum Pupulin \& Mora-Ret., Selbyana 18(1): 7. 1997. Costa Rica: San José; Alajuelita, en lomas sobre Barrio La Mora, ca. $1850 \mathrm{~m}, 17$ jul 1995, F. Pupulin \& M. Flores 315 (HT: USJ, IT: USJ).

31. Malaxis talamancana Dressler, Novon 5(2): 142. 1995. Costa Rica: San José; Cordillera de Talamanca, Pérez Zeledón, Villa Mills, ca. 3000 m, 28 mar 1993, R.L. Dressler \& D.E. Mora s.n. (HT: USJ-48484).

32. Masdevallia fonsecae W. Königer, Arcula 2: 35. 1994. Costa Rica: Puntarenas; Coto Brus, nördlich von San Vito, 900-1000 m, Fundort entdeckt von Sr. W. Fonseca Arce, geblüht in Kultur in München, Apr 1997, W. Königer WK-34 (HT: M; IT: K, USJ, Herb. Königer).

33. Maxillaria atwoodiana Pupulin, Orchidee (Hamburg) 54(5): 563. 2003. Costa Rica: San José; Dota, El Brujo, ca. 400 m, collected by L. Elizondo, Dec 1999, flowered in cultivation at El Silencio de Savegre, 14 Jan 2000, F. Pupulin 1913 (HT: USJ).

34. Maxillaria cedralensis J.T. Atwood \& MoraRet., Selbyana 18(1): 31. 1997. Costa Rica: San José; Dota, Cedral, 2000 m, abr 1990, D.E. Mora \& B-357 s.n. (HT: USJ-31579).

35. Mesospinidium horichii Bock, Orchidee (Hamburg) 42(5): 232. 1991. Costa Rica: Heredia; Sarapiquí, Atlantic rainforest, Sarapiquí junglas, sep gulch near Cariblanco, 800 m, 1988, C.K. Horich s.n. (HT: Sammlung Bock). USJ: Dibujos y fotos del HT.

36. Myoxanthus vittatus Pupulin \& M.A. Blanco, Lankesteriana 2: 16. 2001. Costa Rica: San José; Pérez Zeledón, El Brujo, cerca del Río División, 450 m, 30 jun 2000, floreció en cultivo en el Jardín Botánico Lankester, 25 mayo 2004, M.A. Blanco .... (LT, designado aquí: USJ en líquido. El holotipo nunca fue enviado a USJ ni a otro herbario; de la planta original se preparó este segundo ejemplar.) = Pleurothallis grammata Dressler, Lankesteriana 3: 28. 2002. Echinella vittata (Pupulin \& M.A. Blanco) Pupulin, Lankesteriana 4: 17. 2002 (nom. nud.). Echinosepala vittata (Pupulin \& M.A. Blanco) C.O. Morales \& N. Villal., comb. nova. Esta combinación nueva es necesaria para actualizar la nomenclatura de este taxon, según el análisis de cuatro secuencias de ADN realizado por Pridgeon \& Chase (2001).

37. Odontoglossum hortensiae R.L. Rodr., Orquídea (Méx.) 7(3): 145. 1979. Costa Rica: Cartago; cult. L. Glicenstein, 15 mayo 1978, R.L. Rodríguez 1560 (HT: USJ, IT: AMES, F). Lemboglossum hortensiae (R.L. Rodr.) Halb., Orquídea (Méx.) 9: 349. 1984. Rhynchostele hortensiae (R.L. Rodr.) Soto Arenas \& Salazar, Orquídea (Méx.) 13(1-2): 149. 1993.

38. Oerstedella $\mathrm{x}$ monteverdensis Pupulin \& Hágsater, Lankesteriana 8: 32.2003 [O. endresii (Rchb.f.) Hágsater x O. exasperata (Rchb.f.) Hágsater]. Costa Rica: Puntarenas; Monteverde, Cerro Plano, finca Beeche, $10^{\circ} 19^{\prime} 13^{\prime \prime N}$, $84^{\circ} 48^{\prime} 35^{\prime \prime} \mathrm{W}, 1550 \mathrm{~m}$, collected by G. Barboza, 1998, flowered in cultivation at the Orchid Garden in Monteverde, 2 Jun 2001, F. Pupulin 3216 (HT: USJ).

39. Ornithocephalus castelfrancoi Pupulin, Lindleyana 15(1): 27. 2000. Costa Rica: San José; Dota, eastern slopes of Cerro Nara, $740 \mathrm{~m}$, $9^{\circ} 28^{\prime} 20^{\prime \prime} \mathrm{N}, 8^{\circ} 00^{\prime} 25^{\prime \prime} \mathrm{W}, 14$ Jan 1999, F. Pupulin et al. 1069 (HT: USJ).

40. Ornithocephalus grex-anserinus Dressler \& Mora-Ret., Novon 7(2): 120. 1997. Costa Rica: Alajuela; Sarapiquí, alrededores de Laguna María Aguilar, $10^{\circ} 18^{\prime} \mathrm{N}, 84^{\circ} 11^{\prime} \mathrm{O}, 500-700 \mathrm{~m}, 5$ mayo 
1992, D.E. Mora-Retana et al. s.n. (HT: USJ49975).

41. Ornithocephalus montealegreae Pupulin, Orchids 71(11): 1017. 2002. Costa Rica: Cartago; Turrialba, Moravia de Chirripó, Platanillo, along the Quebrada Tsipirí, 9 $49.4^{\prime} \mathrm{N}, 83^{\circ} 24.5^{\prime} \mathrm{W}$, 1090 m, 3 May 2002, F. Pupulin et al. 3607 (HT: USJ, IT: CR, USJ en líquido). Costa Rica: Limón; Guápiles, San Valentín, area between Río Costa Rica and Río Toro Amarillo, ca. $10^{\circ} 10^{\prime} \mathrm{N}, 83^{\circ} 50^{\prime} \mathrm{W}, 600 \mathrm{~m}$, Apr 2000, flowered in cultivation, 20 May 2002, A. Acuña 9 (PT: USJ en líquido).

42. Paphinia subclausa Dressler, Novon 7(2): 121. 1997. Costa Rica: Alajuela; Reserva Juan Castro Blanco, 900 m, ago 1989, D.E. Mora s.n. (HT: USJ-31027).

43. Pleurothallis bitumida Luer, Lindleyana 11(2): 70, f.11. 1996. Costa Rica: Heredia; Sarapiquí, Parque Nacional Braulio Carillo, El Ceibo Station, $500 \mathrm{~m}, 23 \mathrm{Nov}$ 1990, S. Ingram \& $\mathrm{K}$. Ferrell 747 (HT: CR, IT: AMES, F, K, MO, SEL). Un isotipo fue robado de USJ ca. 1995.

44. Pleurothallis blancoi Pupulin, Caesiana 15: 1. 2000. Costa Rica: Puntarenas; Monteverde, Finca San Gerardo, 1300 m, collected by M. Blanco, 23 Jan 1999, flowered in cultivation at Jardín Botánico Lankester, 27 Jun 2000, F. Pupulin 2434 (HT: USJ, IT: SEL).

45. Pleurothallis ingramii Luer, Lindleyana 11(2): 81, f.18. 1996. Costa Rica: Limón; Pococí, Parque Nacional Braulio Carrillo, Carrillo Station, Quebrada González, 650 m, 15 Nov 1990, S. Ingram \& K. Ferrell 697 (HT: CR, IT: MO, SEL). Un isotipo fue robado de USJ ca. 1995. = Stelis ferrelliae Pridgeon \& M.W. Chase, Lindleyana 17(2): 99. 2002, non S. ingramii Luer.

46. Pleurothallis montezumae Luer, Lindleyana 11(2): 83, f.20. 1996. Costa Rica: Alajuela; Upala, Bijagua, Finca Montezuma, ladera sur de Cerro Montezuma, 600 m, 18 jul 1993, G. Herrera 6280 (HT: K, IT: CR). Un isotipo en USJ.

47. Pleurothallis tintinnabula Luer, Lindleyana 11(2): 94, f.27. 1996. Costa Rica: Puntarenas ["Guanacaste"] on border with Alajuela, Monteverde Forest Reserve, near TV towers at 17001750 m, 24 Jun 1989, J.T. Atwood 89-235 (HT:
SEL). Un isotipo en USJ sin flores, pero con restos de 14 inflorescencias; además, los datos de localidad no coinciden exactamente entre el ejemplar y la publicación. Puntarenas; Monteverde, $10^{\circ} 18^{\prime} \mathrm{N}, 84^{\circ} 48^{\prime} \mathrm{W}, 1600-1700 \mathrm{~m}, 7$ Jun 1989 (fl), J.T. Atwood 89-132 (PT: USJ). Stelis tintinnabula (Luer) Pridgeon \& M.W. Chase, Lindleyana 16(4): 267. 2001.

48. Ponthieva brenesii Schltr., Repert. Spec. Nov. Regni Veg. Beih. 19: 165. 1923. Costa Rica: Alajuela; "Bois humides à San Pedro de San Ramón", 1200 m, jul 1921, A.M. Brenes 83 (LT: $\mathrm{CR}$, designado por Barringer, Fieldiana, Bot., n.s., 17: 18. 1986). USJ: Fotocopia del LT ex CR. = Ponthieva maculata Lindl., Ann. Mag. Nat. Hist. 15: 385. 1845.

49. Prosthechea joaquingarciana Pupulin, Selbyana 22(1): 19. 2001. Costa Rica: Heredia; Varablanca, ca. $2000 \mathrm{~m}$, collected by Zayda Rodríguez, 1997, flowered in cultivation at El Roble de Alajuela, 2 Dic 1999, F. Pupulin 1865 (HT: USJ). Costa Rica; "without collection data, a plant flowered at the Orchid Exhibition of Alajuela, 29 Oct 1999", F. Pupulin 1707 (PT: USJ).

50. Prosthechea neglecta Pupulin, Selbyana 22(1): 21. 2001. Costa Rica: San José; Dota, San Pedro, ca. $1900 \mathrm{~m}$, flowered in cultivation at Gaia Botanical Garden in Quepos, May 1999, M. Flores s.n. (HT: USJ-78081).

51. Prosthechea tardiflora Mora-Ret. ex Pupulin, Lankesteriana 3: 23, f.4. 2002. Costa Rica: Guanacaste; Santa Cruz, from Juan Díaz toward Vista al Mar, 500-600 m, collected by R. Cascante A., 1992, flowered in cultivation at Tambor de Alajuela, 15 Jan 2001, F. Pupulin 2806 (HT: USJ). Alajuela; Palmares, orillas del Río Rastrojos, en floración el 26 febr 1994, W. Barahona s.n. (PT: USJ-51074, con un duplicado en líquido: USJ-57361). Loc. cit., a orillas del Río Jesús María, marzo de 1994, D.E. Mora s.n. (PT: USJ57851).

52. Scaphyglottis geminata Dressler \& Mora-Ret., Orquídea (Méx.) 13(1-2): 192. 1993. Costa Rica: Cartago; Jiménez, Taus, 20 ago 1984, D.E. Mora \& R.L. Dressler 31 (HT: USJ-32348, IT: USJ32347 y 32350$)$. Un isotipo también en CR.

53. Schiedeella dressleri Szlach., Fragm. Florist. 
Geobot. 41(2): 855. 1996. Costa Rica: San José; Aserrí, Tarbaca, ca. 1800 m, 25 mar 1993, R.L. Dressler et al. s.n. (HT: Herb. Szlachetko en líquido). El ejemplar seco original se halla en USJ (IT: USJ-48448); flores enviadas por R.L. Dressler a D. Szlachetko representan el holotipo.

54. Sigmatostalix adamsii Dodson, Selbyana 2(1): 54. 1977. Ecuador: Pichincha; Santo Domingo, behind Hotel Zaracay, 675 m, 15 Jun 1967, C.H. Dodson et al. 3705 (HT: SEL). USJ: Fotocopia del holotipo ex SEL.

55. Sigmatostalix cardioglossa Pupulin, Harvard Pap. Bot. 8(1): 38, f.1. 2003. Costa Rica: San José; Pérez Zeledón, San Ramón Norte, trail to Cerro Pelón, $9^{\circ} 25^{\prime} \mathrm{N}, 83^{\circ} 44^{\prime} \mathrm{W}$, ca. $1050 \mathrm{~m}$, collected by J. Cambronero and F. Pupulin, flowered in cultivation [at Jardín Botánico Lankester, Cartago], 3 Dec 2001, F. Pupulin 3499 (HT: USJ).

56. Sigmatostalix pseudounguiculata Pupulin \& Dressler, Lindleyana 15(1): 27. 2000. Costa Rica: San José; Dota, San Joaquín, collected by M. Flores, 25 Nov 1996, flowered in cultivation at Jardín Botánico Lankester, Paraíso de Cartago, 6 Jan 1998, F. Pupulin 497 (HT: USJ, IT: CR, SEL). En USJ sólo un isotipo hasta ahora.

57. Sigmatostalix savegrensis Pupulin, Harvard Pap. Bot. 8(1): 55, f.12. 2003. Costa Rica: San José; Pérez Zeledón, Savegre, Peor Es Nada, margen izquierda de Quebrada Misteriosa, 9 $31^{\prime} 10^{\prime \prime} \mathrm{N}$, 8351'30"W, 1700 m, 4 ago 1994, G. Herrera et al. 7282 (HT: USJ). Loc. cit., 2 ago 1994, G. Herrera et al. 7220 (PT: USJ). Loc. cit., Savegre Arriba, márgenes del Río Savegre, 1300-1600 m, leg. E. Víquez, jun 2000, floreció en cultivo, 10 dic 2002, R.A. Valverde 225 (PT: USJ en líquido).

58. Sobralia chrysostoma Dressler, Orchids 70: 750 2001. Costa Rica: Alajuela; lava beds near Tabacón Lodge, 16 Jul 1999, R.L. Dressler 6166 (HT: MO, IT: USJ)

59. Sobralia dissimilis Dressler, Novon 5(2): 142 1995. Costa Rica: Puntarenas; Monteverde Reserve, TV towers, $1750 \mathrm{~m}, 26$ Jun 1989, J.T. Atwood 89-243 (HT: MO, IT: CR, FLAS, SEL). Un isotipo en USJ; en años pasados las flores fueron dañadas por insectos.
60. Sobralia doremiliae Dressler, Novon 5(2): 142. 1995. Costa Rica: Cartago; Paraíso, Orosi, Parque Nacional Tapantí, 12 nov 1993, D.E. Mora \& R.L. Dressler s.n. (PT: USJ-50001). Espécimen anotado como paratipo por R.L. Dressler.

61. Sobralia kerryae Dressler, Orchid Digest 62(2): 90, f.1. 1998. Costa Rica: Puntarenas; Las Nubes de Santa Elena, WSW of San Isidro, ca. 1150 m, 22 Mar 1995, R.L. Dressler 6178 (HT: MO). Un isotipo en USJ.

62. Sobralia purpurea Dressler, Novon 10(3): 200. 2000. Costa Rica: San José; Desamparados, Casamata, km 39, Carretera Interamericana Sur, entrada a San Cristóbal Norte, 1800 m, jun 1990, floreció en cultivo, 19 abr 1993, D.E. Mora \& C. Quirós s.n. (HT: USJ-48911, dos láminas)

63. Sobralia quinata Dressler, Lankesteriana 6: 27. 2003. Costa Rica: Cartago; Turrialba, Moravia de Chirripó, Quebrada Tsipirí, 948’N, 8323 'W, 1090 m, 26 abr 2002, F. Pupulin et al. 3644 (HT: USJ).

64. Stellilabium anacristinae Pupulin, Harvard Pap. Bot. 8(1): 30, f.1. 2003. Costa Rica: Puntarenas; Aguirre, road between Quepos and San Marcos de Tarrazú, $9^{\circ} 35^{\prime} \mathrm{N}, 84^{\circ} 06^{\prime} \mathrm{W}, 830 \mathrm{~m}, 16$ Febr 2002, F. Pupulin et al. 3517 (HT: USJ, IT: CR, USJ). Dos isotipos en USJ, además del holotipo.

65. Stellilabium boylei J.T. Atwood, Icon. P1. Trop. 14: t.1392. 1989. Costa Rica: Puntarenas; Monteverde, $10^{\circ} 18^{\prime} \mathrm{N}, 84^{\circ} 49^{\prime} \mathrm{W}, 1400 \mathrm{~m}, 25 \mathrm{Mar}$ 1989, J.T. Atwood \& B. Boyle 28-89 (HT: CR, IT: USJ).

66. Stellilabium erratum Dresssler, Lankesteriana 2: 11. 2001. Costa Rica: Cartago; Paraíso, Orosi, Parque Nacional Tapantí, Sendero Oropéndola, 1350 m, 2 nov 1992, J.T. Atwood \& D.E. Mora 4202 (HT: USJ). Un ejemplar seco más dos flores en líquido.

67. Stellilabium kewense Pupulin, ined., Costa Rica: Cartago; El Guarco, Casamata, 9०46'36"N, 8359’16"W, 1913 m, 26 mar 2002, F. Pupulin et al. 3557 (HT: USJ en líquido).

68. Stellilabium lateritium Pupulin, ined., Costa Rica: Cartago; Paraíso, Orosi, Purisil, road to Monte Sky, ca. $9^{\circ} 47^{\prime} \mathrm{N}, 83^{\circ} 50^{\prime} \mathrm{W}, 1550-1600 \mathrm{~m}, 29$ Sept 2002, F. Pupulin et al. 4186 (HT: USJ en lí- 
quido).

69. Stellilabium smaragdinum Pupulin \& M.A. Blanco, Lankesteriana 5: 28. 2002. Costa Rica: Cartago; El Guarco, La Chonta, turbera, $9^{\circ} 42^{\prime} 00^{\prime \prime N}, 83^{\circ} 56^{\prime} 20^{\prime \prime} \mathrm{W}, 2400 \mathrm{~m}, 17$ jul 2001, M. Blanco et al. 1965 (HT: USJ). Un ejemplar seco más flores en alcohol.

70. Stellilabium tsipiriense Pupulin, Harvard Pap. Bot. 8(1): 30, f.2. 2003. Costa Rica: Cartago; Turrialba, Moravia de Chirripó, Quebrada Tsipirí, $9^{\circ} 48^{\prime} \mathrm{N}, 83^{\circ} 23^{\prime} \mathrm{W}, 1090 \mathrm{~m}, 26$ abr 2002, F. Pupulin et al. 3610 (HT: USJ, IT: CR, SEL, USJ). Sólo un IT en USJ. Loc. cit., Platanillo, $9^{\circ} 49.4^{\prime} \mathrm{N}, 83^{\circ} 24.5^{\prime} \mathrm{W}, 1090 \mathrm{~m}$, along the Quebrada Tsipirí, 3 May 2002, F. Pupulin et al. 3726 (PT: USJ).

71. Telipogon storkii Ames \& C. Schweinf. ssp. magnificus Dodson \& Escobar, Orquideología 17(2): 128. 1987. Costa Rica: Heredia; above San José de la Montaña, passing Porrosatí, "Paso Llano", 2300 m, 25 oct 1984, R. Escobar \& R. Vega 3590 (HT: JAUM, IT: ANT, COL, CR, MO, RPSC, USJ). En USJ, además, una fotografía en colores de la inflorescencia (USJ-71182), tomada antes de ser preparado el ejemplar tipo; esta foto estuvo muchos años en poder de Dora E. Mora, en la Universidad de Costa Rica. Basiónimo: T. storkii Ames \& C. Schweinf., Sched. Orch. 10: 101. 1930.

72. Trichocentrum candidum Lindl., Edwards's Bot. Reg. 29: Misc. 9. 1843. Guatemala; s.d., G. Skinner s.n. (K). USJ: Fotografía en colores del tipo ex K.

73. Trichocentrum capistratum Linden \& Rchb.f., Gard. Chron. 1257. 1871. Costa Rica; sin localidad exacta, floreció en el jardín de Linden, Bélgica, 23 sept 1868, Wallis s.n. (HT: W). USJ: Dos fotocopias del tipo ex W.

74. Trichocentrum costaricense Mora-Ret. \& Pupulin, Selbyana 15(2): 94. 1994. Costa Rica: San José; Pérez Zeledón, San Isidro del General, "florece en mayo", C. Horich s.n. (LT, designado aquí: USJ-57468, una flor en líquido). No se halló ningún ejemplar tipo en USJ; hasta ahora sólo existen dos frascos, cada uno con una flor de esta especie. La localidad en el LT no es segura; compárense los datos del LT con los del holotipo (Alajuela; San Carlos Plain, near Ciudad Quesada, 1987, C.K. Horich s.n.).

75. Trichocentrum cymbiglossum Pupulin, Lindleyana 9(1): 51. 1994. Costa Rica: Alajuela; Grecia ["San Carlos"], Laguna Bosque Alegre, 750 m, mayo 1989, F. Pupulin 5 (HT: USJ, IT: Herb. Pupulin).

76. Trichocentrum dianthum Pupulin \& Mora-Ret., Selbyana 15(2): 90. 1994. Costa Rica: San José; Pérez Zeledón, Las Nubes de Quizarrá, 1000 m, floreció en cultivo, mar 1989, J. Cambronero s.n. (HT: USJ-38972).

77. Trichocentrum estrellense Pupulin \& J.B. García, Lindleyana 10(3): 195, f.8. 1995. Costa Rica: Cartago; El Guarco, in valleys under Palo Verde, along a minor tributary of Río Reventazón, 1250 m, 30 Apr 1992, F. Pupulin 209 (HT: USJ, IT: Herb. Pupulin). En realidad, no hay ningún ejemplar de esta especie en USJ, por lo que será necesario designar un neotipo.

78. Trichocentrum maculatum Lindl., Orchid. Linden. 24, No. 127. 1846. Colombia: Río Hacha, Sierra de Santa Marta, junto al Río San Antonio, 1300 m, 1844, Linden 1666 (HT: K, IT: BR, P, W). USJ: Fotocopia del tipo ex AMES. $=$ Trichocentrum pulchrum Poepp. \& Endl., Nov. Gen. Sp. P1. 2: 11, t.115. 1836.

79. Trichocentrum obcordilabium Pupulin, Novon 8(3): 238, f.1. 1998. Ecuador: Morona-Santiago; San Juan Bosco, 1600 m, 16 Febr 1993, collected by J. Portilla, flowered in cultivation, $6 \mathrm{Jul}$ 1996, F. Pupulin 285 (HT: SEL, IT: MO, QC$\mathrm{NE)}$. Un isotipo en USJ.

80. Trichocentrum pfavii Rchb.f., Gard. Chron., n.s. 16: 70. 1881. Panamá: Chiriquí, 19 nov 1880, $R$. Pfau 60 (HT: W). USJ: Ilustración del tipo ex W (dos láminas).

81. Trichocentrum pfavii Rchb.f. ssp. dotae Pupulin, Selbyana 22(1): 23. 2001. Costa Rica: San José; Dota, Santa María, road to San Joaquín, collected by A. Flores, Apr 1999, flowered in cultivation at Gaia Botanical Garden (Quepos), 11 Dec 1999, F. Pupulin 1871 (HT: USJ). San José; Dota, San Marcos, camino a San Joaquín, 1410 m, oct 1989, F. Pupulin \& M. Flores 6 (PT: USJ). 
82. Trichocentrum pinelii Lind1., Gard. Chron.: 772. 1854. Brasil: Rio de Janeiro; [sin localidad exacta], s.d., Pinel s.n. (HT: K). USJ: Foto en colores del holotipo ex K. = Trichocentrum fuscum Lindl., Edwards's Bot. Reg. 23: t. 1951. 1837.

83. Trichocentrum viridulum Pupulin, Novon 8: 285. 1998. Colombia: Santander; Charalá, Virolín, 1900 m, collected in Mar 1990, flowered in cultivation at Colomborquídeas, 24 Aug 1996, F. Pupulin 388 (HT: SEL, IT: MO). Un isotipo en USJ.

84. Trichocentrum wagneri Pupulin, Lindleyana 10(3): 203, f.14. 1995. Brasil; without collection data, introduced by A. Seidel, flowered in cultivation by A. Wagner in Santa Margherita, Italy, 25 Sept 1990, F. Pupulin 289 (HT: SEL). Un isotipo en USJ.

85. Trichopilia x ramonensis J. García \& Mora-Ret. ex C.O. Morales, Lankesteriana 5: 18. 2002. Costa Rica: Alajuela; San Ramón, San Rafael, Berlín, $10^{\circ} 03^{\prime} \mathrm{N}, 84^{\circ} 28^{\prime} \mathrm{O}$, floreció en cultivo, mar 1991, Luis Acosta s.n. (HT: USJ-57879, en líquido). Alajuela; San Ramón, Cataratas, 19 mar 1992, L. Acosta s.n. (PT: USJ-44878). Alajuela; San Ramón [sin localidad exacta], febr 1987, floreció en cultivo, mar 1990, L. Acosta s.n. (PT: USJ-31985).

86. Trisetella lasiochila Pupulin, Lindleyana 15(1): 30. 2000. Costa Rica: Cartago; El Guarco; San Isidro, Casamata, km 39, Carretera Interamericana sur, ca. 1800 m ["Tejar de San Carlos"], collected by A. Flores, 3 Febr 1991, flowered in cultivation at Jardín Botánico Lankester, Paraíso de Cartago, 1400 m, 17 May 1999, F. Pupulin 1463 (HT: USJ, IT: SEL en líquido).

\section{POACEAE}

1. Diandrolyra bicolor Stapf, Bull. Misc. Inform. Kew 1906: 204. 1906. "Cultivated: Native country unknown. Raised at Kew from seeds communicated by Messers. Sander \& Son.", Sander 100-03 (HT: K). USJ: Fotocopia más ilustraciones del holotipo ex K. Ahora sabemos que la especie habita en Brasil; en el sur de este país fue redescubierta en 1972 por Thomas Söderstrom (Söderstrom \& Zuloaga 1985).
2. Digitaria pulchella Griseb., Cat. Pl. Cub.: 231. 1866. Cuba: Oriente; Yunque de Baracoa, on vertical cliffs, 8 Jun 1856, C. Wright 3448 (HT: GOET, IT: GH, LE, MO, NY, US). USJ: Fotocopia de un isotipo ex B. Mniochloa pulchella (Griseb.) Chase, Proc. Biol. Soc. Wash. 21: 186. 1908.

3. Ekmanochloa aristata Hitchc., Man. Grasses W. Ind.: 377, f.343B. 1936. Cuba: Oriente; collected in carrascales-pinales, between Taco and Nibujon, very rare, 4 Dec 1914, E.L. Ekman 3729 (HT: US, IT: S). USJ: Fotocopia de un isotipo ex B.

4. Ekmanochloa subaphylla Hitchc., Man. Grasses W. Ind.: 375, f.343A. 1936. Cuba: Oriente; Sierra de Nipe, collected on overhanging limestone rocks of Loma Picote, 500 m, 2 Nov 1922, E.L. Ekman 9870 (HT: US, IT: MO, NY, US). USJ: Fotocopia de un isotipo ex B.

5. Olyra lancifolia Mez, Notizbl. Bot. Gart. Mus. Berlin-Dahlem 7(63): 45. $1917 . \quad$ Brasil: Amazonas; ad flumen Jurua prope Fortaleza, oct 1901, E.H. Ule 5951 (HT: B, IT: HBG). US J : Fotocopia del holotipo ex B. Agnesia lancifolia (Mez) Zuloaga \& Judz., Novon 3(3): 307, f.1. 1993.

6. Olyra pineti C. Wright ex Griseb., Mem. Amer. Acad. Arts, n.s. 8: 532. 1862. Cuba: prope Villa Monte Verde, inter pinorum folia dejecta, 22 aug 1859, C. Wright 1536 (HT: GOET, IT: GH, MO, NY, US). USJ: Fotocopia de un isotipo ex B. Lithachne pineti (C. Wright ex Griseb.) Chase, Proc. Biol. Soc. Wash. 21: 182. 1908.

7. Olyra urbaniana Mez, Notizbl. Bot. Gart. Berlin-Dahlem 7(63): 47. 1917. Trinidad \& Tobago: Tobago; in silvis primaevis montis Morne d'Or, s.d., H. von Eggers 5841 (HT: B, fragmento US). USJ: Fotocopia del holotipo ex B. = Strephium guianense Brongn., Bull. Soc. Bot. France 7: 470. 1860. Raddia guianensis (Brongn.) Hitchc., Man. Grasses W. Ind.: 373. 1936.

8. Piresia leptophylla Söderstr., Brittonia 34(2): 206. 1982. Brasil: Bahía; Una, Fazenda Itapororoca, ca. $10 \mathrm{~km} \mathrm{~S}$ of Una, ca. $2 \mathrm{~km}$ from ocean, sea level, 26 May 1976, T. Söderstrom et al. 2225 (HT: CEPEC, IT: B, CANB, DD, F, K, L, LE, MO, NY, P, PE, PRE, RB, SI, TNS, US). 
USJ: Fotocopia del isotipo ex B.

9. Sucrea monophylla Söderstr., Brittonia 33(2): 200. 1981. Brasil: Bahía; Itabuna, Ferradas, Fazenda Aberta Grande, ca. 14\%47'21"S, 39¹6'36"W, 16 ene 1968, C. Calderón 2045 (HT: CEPEC, IT: K, LE, MO, NY, P, RB, US). USJ: Fotocopia de un isotipo ex B.

\section{SMILACACEAE}

1. Smilax angustiflora A. DC., Monogr. Phan. 1: 67. 1878. Costa Rica: Heredia; Alto de la Cruz, Azarí, jun 1857, C. Hoffmann 575 (HT: B). USJ: Foto del holotipo. = Smilax mollis Humb. \& Bonpl. ex Willd.

2. Smilax candelariae A. DC., Monogr. Phan. 1: 70. 1878. Costa Rica: San José; Acosta, Candelaria, 16 Jun 1857, C. Hoffmann s.n. (HT: B). USJ: Foto del holotipo ex F. = Smilax mollis Humb. \& Bonpl. ex Willd.

3. Smilax caudata Lundell, Wrightia 3(8): 162. 1966. Guatemala: Alta Verapaz; on Coban Road, between Chiracte and Chapultepec Farm, km 285/286, 24 May 1964, E. Contreras 4783 (HT: LL, IT: MO). USJ: Fotocopia del isotipo ex MO. = Smilax domingensis Willd.

4. Smilax domingensis Willd., Sp. Pl. (ed. quarta) 4(2): 783. 1806. República Dominicana ("Habitat in St. Domingo, Porto-rico"), s.d., Richard s.n. USJ: Fotocopia del holotipo ex B.

5. Smilax gymnopoda Apt, Repert. Spec. Nov. Regni Veg. 18: 401. 1922. México: Veracruz; in Dickichten nahe Jalapa in einer Höhe von 1200 m, 14 Apr 1899, Pringle 8130 [LT: GH, designado por Ferrufino en Ferrufino \& Gómez Laurito (2004: 17), ILT: BM, BR, F, G, GH, K, MO, NY, P]. USJ: Foto de un isolectotipo. = Smilax mollis Humb. \& Bonpl. ex Willd.

6. Smilax kunthii Killip \& C.V. Morton, Publ. Carnegie Inst. Wash. 461: 269. 1936. Ecuador; H. Ruiz \& J. Pavón s.n. (ST: B). USJ: Foto del tipo ex B. = Smilax domingensis Willd.

7. Smilax mollis Humb. \& Bonpl. ex Willd., Sp. Pl. 4: 785. 1806. México: Veracruz; Jalapa, s.d., Humboldt \& Bonpland s.n. (HT: P). USJ: Fotocopia del holotipo.

8. Smilax munda Killip \& C.V. Morton, Publ. Carnegie Inst. Wash. 461: 265. 1936. British Honduras; on the bank of the Rio Grande, 23 Jul 1933,
W.A. Schipp 1181 (HT: F, IT: K, MICH, MO). USJ: Foto del holotipo. = Smilax spinosa Mill., Gard. Dict. ed. 8, no. 8. 1768.

9. Smilax panamensis Morong, Bull. Torrey Bot. Club 21: 441. 1894. Panamá; Gatún Station, s.d., Hayes 63 (LT: NY, designado por Killip \& Morton, Publ. Carnegie Inst. Wash. 461: 275. 1936). USJ: Fotocopia del lectotipo y de un isolectotipo.

10. Smilax spinosa Mill. var. compta Killip \& C.V. Morton, Publ. Carnegie Inst. Wash. 461(12): 264. 1936. Panamá: Panamá; collected on dry limestone in forests around Alhuela, Chagres Valley, 30-100 m, 12-15 May 1911, H. Pittier 3487 (HT: US). USJ: Fotocopia de un isotipo ex NY.

11. Smilax subpubescens A. DC., Monogr. Phan. 1: 69. 1878. México: Veracruz: región de Orizaba, San Cristóbal, 1865-1866, M. Bourgeau 2578 (HT: P, IT: K). USJ: Foto del holotipo ex F.

12. Smilax vanilliodora Apt, Repert. Spec. Nov. Regni Veg. 18: 416. 1922. Costa Rica: Cartago; Turrialba, Hacienda El Guayabo, 600-700 m, s.d., J. Gómez Álvarez s.n. (HT: B). USJ: Dos fotocopias del holotipo ex F.

13. Smilax velutina Killip \& C. Morton, Publ. Carnegie Inst. Wash. 461(12): 283. 1936. México: Chiapas; Finca Mexiquito, jul 1913, C. Purpus 6930 (HT: US, IT: BM, F, G, MO, NY). USJ: Fotocopia de un isotipo ex MO.

14. Smilax venosa Lundell, Wrightia 3(8): 165. 1966. México: Chiapas; Pinabeto, near Motozintla, 2585 m, 7 May 1945, E. Matuda 5426 (HT: LL). USJ: Foto de un isotipo ex F y fotocopia de un isotipo ex MO. $=$ Smilax subpubescens A. DC.

\section{LITERATURA CITADA}

Ammann, K. 1986. Die Bedeutung der Herbarien als Arbeitsinstrument der botanischen Taxonomie; zur Stellung der organismischen Biologie heute. Bot. Helv. 96(1): 109-132.

Andrade, A.G. de, F. Atala, Emmerich, Margarete, Travassos, P. Odette \& H.F. Martins. 1961. Os tipos das plantas vasculares do Herbario do Museu Nacional I. Bol. Mus. Nac. (Rio Janeiro) n. ser., Bot. 28: 1-22.

Clark, J.R., B.K. Holst \& L.E. Skog. 2003. An annotated checklist of Gesneriaceae type specimens in the Marie Selby Botanical Gardens Herbarium (SEL). Selbyana 24(2): 119-140.

Cronquist, A. 1992. An Integrated System of Classification of Flowering Plants. Columbia University Press. 
$1262 \mathrm{p}$.

Davies, A.M.R., Bodensteiner, P., Pillukat, A. \& Grau, J. 2002. INFOCOMP - the Compositae Types digital imaging project in Munich. Sendtnera 8: 9-20.

Ferrufino, Lilian \& Gómez Laurito, J. 2004. Estudio morfológico de Smilax L. (Smilacaceae) en Costa Rica, con implicaciones sistemáticas. Lankesteriana 4(1): 5-36.

Gerlach, G. 1994. Kefersteinia retanae, una nueva orquídea de Costa Rica. Brenesia 41-42: 99-106.

Grayum, M.H. 2003. Arecaceae. In: B.E. Hammel, M.H. Grayum, C. Herrera \& N. Zamora (eds.). Manual de Plantas de Costa Rica 2. Missouri Botanical Garden, INBio, Museo Nac. de Costa Rica. p. 201-293.

Greuter, W., J. McNeill, F.R. Barrie, H.M. Burdet \& V. Demoulin. 2000. International Code of Botanical Nomenclature (Saint Louis Code). XVI International Botanical Congress, St. Louis, 1999. 474 p.

Henderson, A., G. Galeano \& R. Bernal. 1995. Field guide to the palms of the Americas. New Jersey, Princeton Univ. Press. 352 p.

Lobo C., Silvia. 2002. Colección tipo del Herbario Nacional de Costa Rica (CR). III. Revisión y actualización de las monocotiledóneas. Brenesia 57-58: 135- 150.
Morales, C.O. 2002. Notas varias sobre Heliconia rodriguezii (Heliconiaceae) de Costa Rica. Lankesteriana 5: 23-25.

Nilsson, V. \& G. Umaña. 1995. Colección tipo del Herbario Nacional de Costa Rica (CR). I. Liliatae. Brenesia 43-44: 71-89.

Pridgeon, A.M. \& M.W. Chase. 2001. A phylogenetic reclassification of Pleurothallidinae (Orchidaceae). Lindleyana 16(4): 235-271.

Pupulin, F. \& G. Romero-González. 2003. Costa Rican Orchidaceae Types (CROTYPES) digital imaging documentation at AMES, Harvard University. Lankesteriana 7: 11-16.

Roberts, D.L. \& G.J. McInerny. 2003. When is a species extinct? Quantitative inference of threat and extinction from herbarium data. Lankesteriana 7: 17-20.

Ruiz-Boyer, A. \& R. González-Ball. 2002. Colección tipo del Herbario Nacional de Costa Rica (CR). II. Fungi, Lichenes, Bryophyta y Pteridophyta. Brenesia 57-58: 113134.

Söderstrom, T. \& F. Zuloaga. 1985. Diandrolyra tatianae (Poaceae: Olyreae), a new herbaceous Bamboo from Brazil. Brittonia 37(1): 1-5. 